\title{
OPTICAL AND X-RAY IDENTIFICATION OF FAINT RADIO SOURCES IN THE GOODS CDF-S ADVANCED CAMERA FOR SURVEYS FIELD
}

\author{
J. Afonso, ${ }^{1,2}$ B. Mobasher, ${ }^{3,4}$ A. Koekemoer, ${ }^{3}$ R. P. Norris, ${ }^{5}$ and L. Cram ${ }^{6}$ \\ Received 2005 May 5; accepted 2005 September 27
}

\begin{abstract}
We present optical and X-ray identifications for the 64 radio sources in the Great Observatories Origins Deep Survey Chandra Deep Field-South Advanced Camera for Surveys (ACS) field revealed in the Australia Telescope Compact Array 1.4 GHz survey of the Chandra Deep Field-South. Optical identifications are made using the ACS images and catalogs, while the X-ray view is provided by the Chandra X-Ray Observatory $1 \mathrm{Ms}$ observations. Redshifts for the identified sources are drawn from publicly available catalogs of spectroscopic observations and multiband photometric-based estimates. Using this multiwavelength information we provide a first characterization of the faint radio source population in this region. The sample contains a mixture of star-forming galaxies and active galactic nuclei, as identified by their X-ray properties and optical spectroscopy. A large number of morphologically disturbed galaxies are found, possibly related to star formation. In spite of the very deep optical data available in this field, seven of the 64 radio sources have no optical identification to $z_{850} \sim 28 \mathrm{mag}$. Only one of these is identified in the X-ray.
\end{abstract}

Key words: galaxies: active — galaxies: evolution — galaxies: starburst — radio continuum: galaxies

Online material: machine-readable tables

\section{INTRODUCTION}

The Great Observatories Origins Deep Survey (GOODS; Giavalisco et al. 2004) provides deep multiwavelength observations in the Hubble Deep Field-North (HDF-N) and Chandra Deep Field-South (CDF-S). The fields have been imaged by the Hubble Space Telescope (HST), using the Advanced Camera for Surveys (ACS) in four bands (F435W, F606W, F775W, and F850LP). At infrared wavelengths, observations by the Spitzer Space Telescope use the IRAC (3.6-8 $\mu \mathrm{m})$ and MIPS $(24 \mu \mathrm{m})$ instruments. The deepest X-ray surveys currently available have been made in the GOODS fields, using the Chandra X-Ray Observatory and XMM-Newton. The GOODS fields are also targets of many ground-based studies using $4 \mathrm{~m}$ and $8 \mathrm{~m}$ class telescopes, providing imaging and spectroscopic data in optical and nearinfrared bands. The richness and depth of data imply that GOODS will be a foundation for the study of galaxy formation and evolution over the next few years.

Space- and ground-based observations and data acquired up to 2004 are described by Giavalisco et al. (2004). The available spectroscopic redshifts for these fields, combined with the multiwave band imaging data set, have been used to calibrate and determine photometric redshifts of many sources to faint magnitudes (e.g., Mobasher et al. 2004; Wolf et al. 2004).

Deep radio observations of GOODS represent an excellent opportunity to explore a number of unresolved questions regarding radio emission from galaxies, since GOODS sources have a

\footnotetext{
${ }^{1}$ Observatório Astronómico de Lisboa, Faculdade de Ciências, Universidade de Lisboa, Tapada da Ajuda, 1349-018 Lisbon, Portugal; jafonso@oal.ul.pt.

${ }^{2}$ Centro de Astronomia e Astrofísica da Universidade de Lisboa, Lisbon, Portugal.

${ }^{3}$ Space Telescope Science Institute, 3700 San Martin Drive, Baltimore, MD 21218.

${ }^{4}$ Affiliated with the Space Sciences Department of the European Space Agency.

${ }^{5}$ Australia Telescope National Facility, P.O. Box 76, Epping, NSW 1710, Australia.

${ }^{6}$ Australian National University, Canberra, ACT 0200, Australia.
}

plethora of multiwavelength data available for their characterization. In addition, radio data on GOODS sources can, in some situations, resolve uncertainties and/or provide unique insights about the astrophysical processes at play.

It is believed that the decimetric radio emission from galaxies is dominated by the synchrotron emission from electrons accelerated either by shock waves associated with supernovae (and hence, star formation), by processes energized by a massive nuclear object (an active galactic nucleus [AGN]), or by a combination of the two (Condon 1992).

Radio emission associated with AGNs is responsible for the great majority of decimetric sources brighter than about $5 \mathrm{mJy}$. The optical hosts of these sources include massive elliptical galaxies, QSOs, and Seyfert galaxies. Radio selection of sources brighter than a few millijanskys preferentially selects radio-loud AGNs having relatively high radio-to-optical luminosity ratios. AGNs also exist in the submillijansky population; some are distant radio-loud sources, and others are intrinsically low-luminosity radio sources hosted by objects similar to those hosting radioloud AGNs. Observations of the radio emission from AGNs provide information about the existence and properties of central black holes, especially when combined with observations of X-ray power (e.g., Merloni et al. 2003), as well as clues to some properties of the interstellar and intergalactic medium.

Among the samples of radio sources selected at ever fainter submillijansky flux densities, the proportion of AGNs systematically declines in favor of sources energized by star formation (e.g., Windhorst et al. 1985; Georgakakis et al. 1999; Afonso et al. 2005). The optical hosts of these star-forming radio sources include blue galaxies with optical colors, revealing a young stellar population, and extremely red galaxies with evidence for a very high degree of dust extinction. High-resolution optical imaging often reveals disturbed morphologies indicative of interactions and/or merging activity among the submillijansky radio population (e.g., Richards et al. 1998).

There is a tight correlation between the decimetric radio luminosity and the far-infrared (FIR) luminosity of star-forming 
galaxies. In such sources the FIR emission is believed to be thermal emission from dust heated by stellar UV and optical emission. When the extinction is so high that almost all the stellar luminosity is absorbed by dust, the FIR luminosity measures the total UV/ optical power and hence (if the initial mass function is known) the rate of star formation (e.g., Yun et al. 2001). The correlation between FIR and radio luminosity allows the use of radio power observations to determine star formation rates for galaxies that are too faint to be detected as FIR sources. Importantly, decimetric radio emission is free of extinction.

Deep decimetric observations of GOODS fields thus offer astrophysically useful data on black hole properties, star formation processes, properties of the interstellar and intergalactic medium, and the relative densities and evolution of some major galaxy populations. Accordingly, this paper commences the investigation of radio sources in the GOODS CDF-S field, in particular the HST ACS region, which has homogeneous optical, radio, and X-ray coverage. To assist in building a resource for the entire community, optical and X-ray identifications of the sources revealed in the recent Australia Telescope Compact Array (ATCA) $1.4 \mathrm{GHz}$ radio observations (A. Koekemoer et al. 2006, in preparation) are reported promptly. Throughout this paper, and unless otherwise noted, we adopt $H_{0}=70 \mathrm{~km} \mathrm{~s}^{-1} \mathrm{Mpc}^{-1}, \Omega_{M}=0.3$, and $\Omega_{\Lambda}=0.7$.

\section{MULTIWAVELENGTH OBSERVATIONS}

\subsection{Radio Observations}

The CDF-S was observed at radio $(1.4 \mathrm{GHz})$ wavelengths using ATCA. A total of $120 \mathrm{hr}$ of observations were obtained, resulting in a $1.2 \mathrm{deg}^{2}$ area field, covering the CDF-S to a limiting $(1 \sigma)$ sensitivity of $\approx 14 \mu \mathrm{Jy}$ with a beam size of $16^{\prime \prime} .8 \times 6^{\prime \prime} .9$. Source detection provided a catalog of 683 sources, with integrated flux densities between $61 \mu \mathrm{Jy}$ and $170 \mathrm{mJy}$. A. Koekemoer et al. (2006, in preparation) present the observations, data reduction methods, and approach used to construct the radio source catalog.

Within the GOODS CDF-S ACS region, a total of 64 radio sources are found, with $1.4 \mathrm{GHz}$ flux densities between $63 \mu \mathrm{Jy}$ and $20 \mathrm{mJy}$. Figure 1 shows the radio view of this field. This sample and its optical and X-ray properties are given in the present paper.

\subsection{Optical Observations}

The HST ACS observations of the CDF-S taken as part of the GOODS project (Giavalisco et al. 2004) were used. The data release v1.0 includes F435W $\left(B_{435}\right)$, F606W $\left(V_{606}\right)$, F775W $\left(i_{775}\right)$, and F850LP $\left(z_{850}\right)$ reduced, calibrated, stacked, and mosaicked images and the corresponding catalogs, providing photometry of sources detected in the $z_{850}$ band. Combined $B_{435}+V_{606}+i_{775}+$ $z_{850}$ images were made in order to search for faint optical counterparts of radio sources not detected in the single $z_{850}$ band.

\subsection{X-Ray Observations}

The deep Chandra X-Ray Observatory observations of the CDF-S (Giacconi et al. 2002) were used. The integration time amounts to $1 \mathrm{Ms}$, being one of the deepest X-ray observations ever taken.

Initially, the X-ray point-source catalogs from Alexander et al. (2003) were searched for counterparts of radio sources. The signalto-noise ratio sensitivity limits $(\mathrm{S} / \mathrm{N}=3)$ in the $\approx 1 \mathrm{arcmin}^{2}$ region at the aim point are $\approx 5.2 \times 10^{-17}$ and $\approx 2.8 \times 10^{-16}$ ergs $\mathrm{cm}^{-2} \mathrm{~s}^{-1}$ in the $0.5-2.0$ and $2.0-8.0 \mathrm{keV}$ bands, respectively (Alexander et al. 2003).

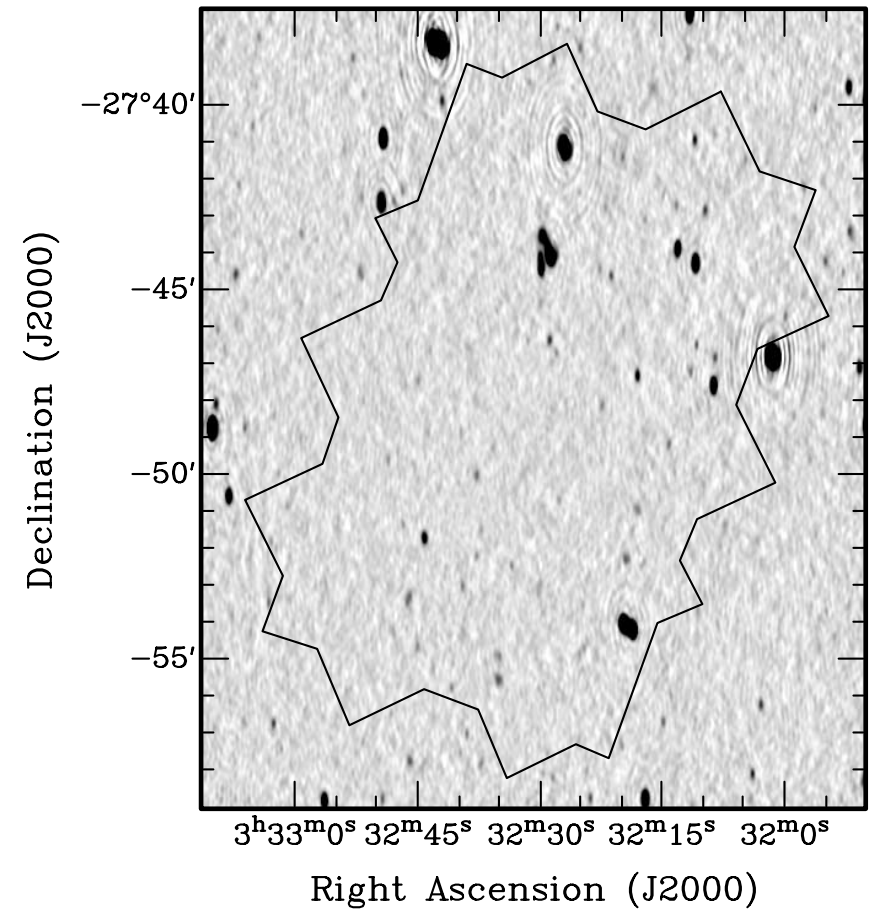

FIG. 1.-ATCA radio $1.4 \mathrm{GHz}$ image of the GOODS CDF-S ACS field (outline). The 64 radio sources detected within this field have integrated $1.4 \mathrm{GHz}$ flux densities ranging from $63 \mu \mathrm{Jy}$ to $20 \mathrm{mJy}$.

The catalogs presented in Giacconi et al. (2002) were also inspected for X-ray detections not reported in Alexander et al. (2003); the different data reduction and source detection methods result in a few faint sources being detected in only one of these works, which is nevertheless still relevant for the current study. However, this imposes some nonuniformity for the reported X-ray characteristics. While the catalogs produced by Alexander et al. (2003) provide counts and fluxes in various bands, covering the $0.5-8.0 \mathrm{keV}$ interval, and provide the effective photon index $\Gamma{ }^{7}$ which is calculated for a power-law model with the Galactic column density taking into account the ACIS quantum efficiency degradation, the catalogs by Giacconi et al. (2002) provide only counts and fluxes in the $0.5-2.0$ and $2.0-10.0 \mathrm{keV}$ bands.

In order to provide comparable quantities for sources detected in these different catalogs, we estimate the X-ray flux in the 2$8 \mathrm{keV}$ band for three sources detected only in the Giacconi et al. (2002) catalogs, using the reported $2.0-10.0 \mathrm{keV}$ flux and assuming a power-law photon index of 1.4 , the average value for the remaining X-ray-detected sources, as mentioned below. Still, the different reduction techniques and assumptions (considering or not the ACIS degradation, for example) between these X-ray catalogs should be noted.

\section{SOURCE IDENTIFICATION}

Optical counterparts of radio sources in the GOODS ACS field were identified using the likelihood ratio method of Sutherland \& Saunders (1992). For each radio source, the optical identification with the highest reliability $(\mathcal{R})$, if above $20 \%$, was taken as the real optical counterpart; if several optical identifications had similar values for $\mathcal{R}$, the various possibilities were considered. Identifications were inspected visually to check for special situations in which the likelihood ratio method would not apply, as in the

\footnotetext{
As described in Alexander et al. (2003).
} 
case of nonindependent sources (either in the radio or in the optical).

The identification of the X-ray counterparts was performed by searching the $3 \sigma$ radio position error region. Visual inspection was used to associate the optical and X-ray identifications.

A few sources require special mention. Source 13 (ATCDFS J033213.28) coincides with two optical sources in the ACS $z_{850}$ image, although the GOODS catalog shows a single identification. SExtractor was used to deblend the two objects and obtain positions and optical magnitudes for both. Source 19 (ATCDFS J033218.62) and source 21 (ATCDFS J033219.62) are 12" apart and display similar radio fluxes (4.8 and $5.9 \mathrm{mJy}$, respectively). They are regarded as being two radio lobes of the same radio galaxy, optically identified with a cD-type galaxy located roughly halfway between the two. Source 55 (ATCDFS J033244.16) is identified with an extended interacting pair of galaxies, whose optical positions lie just outside and beside the radio position error ellipse. This system is considered to be the optical counterpart of the radio emission.

Table 1 presents the final source identifications. The columns display (1) source number; (2) radio source name; (3) $1.4 \mathrm{GHz}$ flux density and associated error; (4) ACS identification; (5) reliability $(\mathcal{R})$ of the ACS identification; (6) distance in arcseconds between the radio and the ACS positions; (7) ACS $z_{850}(\mathrm{AB})$ magnitude; (8) Chandra X-Ray Observatory identification; (9) distance in arcseconds between the radio and the Chandra X-Ray Observatory positions; (10) X-ray flux in the $0.5-2 \mathrm{keV}$ band; and (11) X-ray flux in the $2-8 \mathrm{keV}$ band. Radio sources with no optical counterpart show the $z_{850} 3 \sigma$ upper limit, estimated from the pixel rms $\left(0.0008-0.0015\right.$ counts $\mathrm{s}^{-1}$ pixel $\left.^{-1}\right)$, integrated over a $0.2 \times 0.2$ aperture $(4 \times 4$ ACS pixel box $)$. The reliability calculation for optical identifications assumes each optical detection individually. Whenever a merger or interacting pair of galaxies is identified as the optical counterpart and one of the sources clearly dominates $\mathcal{R}$, we present in Table 1 only the highest value of $\mathcal{R}$, noting that this is a lower limit for the true reliability of the optical system. Sources with an X-ray identification from Giacconi et al. (2002) are identified by their reported source number in that work.

Of the 64 radio sources in the GOODS CDF-S ACS region, two are considered to be radio lobes of the same radio galaxy. For most of the sample, a reliable unique optical counterpart is found, but eight of the radio sources have several possible optical counterparts. Five of these (sources 13, 47, 48, 51, and 55) arise from likely interacting pairs, for which we present in Table 1 data for both members, while the remaining three (sources 41, 52, and 60) are ambiguous identifications, each with two seemingly independent likely optical counterparts. The X-ray detection for sources 41 and 52 suggests which optical counterpart is the real one. Seven $(11 \%)$ of the radio sources have no optical counterpart. One of these, source 42 (ATCDFS J033233.44), is associated with faint X-ray emission reported in the Giacconi et al. (2002) catalog. Figure 2 shows gray-scale images in $B_{435}+V_{606}+i_{775}+z_{850}$, with radio contours and $\mathrm{X}$-ray detections overlaid.

\section{SOURCE CHARACTERIZATION}

Using the deep multiwavelength data available, we provide a first characterization of the radio source population in the GOODS CDF-S ACS field. Several indicators were employed, as described below.

\subsection{X-Ray-to-Optical Flux Ratios}

The X-ray-to-optical flux ratio can provide a first indication of the nature of a galaxy (e.g., Maccacaro et al. 1988; Stocke et al.
1991; Hornschemeier et al. 2003). Here we estimate the X-rayto-optical flux ratio from the relation

$$
f_{\mathrm{X}} / f_{\text {opt }}=\log \left(f_{0.5-2 \mathrm{keV}}\right)+0.4 z_{850}+6.12,
$$

where the X-ray flux is measured in ergs $\mathrm{cm}^{-2} \mathrm{~s}^{-1}$ in the $0.5-$ $2 \mathrm{keV}$ band. This expression results from the X-ray-to-optical flux ratio definition of Stocke et al. (1991), which involves the 0.3-3.5 keV flux and $V$-band optical magnitude. These quantities were converted to $0.5-2 \mathrm{keV}$ flux and $z_{850}$ magnitude assuming a power-law X-ray spectral energy distribution with $\Gamma=1.4$ and a $V-z_{850}$ color of $1.2 \mathrm{mag}$, which are the averages for galaxies in the current sample with detections in these bands. Assuming this expression, AGNs will typically display X-ray-tooptical flux ratios above -1 , while star-forming galaxies tend to display lower values (e.g., Stocke et al. 1991; Schmidt et al. 1998; Shapley et al. 2001; Hornschemeier et al. 2001; Alexander et al. 2002).

\subsection{Radio-to-Optical Flux Ratios}

We also define a radio-to-optical flux ratio as

$$
f_{1.4} / f_{\mathrm{opt}}=\log \left(S_{1.4 \mathrm{GHz}}\right)+0.4 z_{850}-6.56,
$$

where we use the measured radio flux density at $1.4 \mathrm{GHz}$ in millijanskys. Although this is not an AGN/star formation discriminator, since high radio-to-optical flux ratios can be seen in both radio-powerful AGNs or dust-rich galaxies, it is still useful to identify extreme sources in the sample.

\subsection{Spectroscopic Redshifts}

The CDF-S is being intensively studied by several groups. In particular, spectroscopic observations of the optical counterparts of many X-ray sources have been made. The counterparts of the faint radio sources in the GOODS CDF-S ACS field presented here were thus searched for the availability of spectroscopic redshifts from Szokoly et al. (2004), Le Fèvre et al. (2004), Vanzella et al. (2005), and Mignoli et al. (2005). The first reports spectroscopic observations of possible optical counterparts of X-ray sources in the CDF-S down to $R \sim 26$ mag, using the Very Large Telescope (VLT) with the FORS1/FORS2 spectrographs. Le Fèvre et al. (2004) describe the results of the VIMOS VLT Deep Survey around the CDF-S, which includes 784 redshifts in the ACS area down to $I_{\mathrm{AB}}=24 \mathrm{mag}$. Vanzella et al. (2005) present the VLT FORS2 spectroscopic observations of sources in the GOODS CDF-S ACS region. Source selection in that investigation was optimized to detect high-redshift objects in order to take advantage of the characteristics of FORS2 (high throughput and spectral resolution and reduced fringing at red wavelengths), mostly down to $z_{850}=24.5 \mathrm{mag}$. Finally, Mignoli et al. (2005) deal with the VLT FORS1/FORS2 spectroscopic observations of $K_{s}$-selected galaxies from the K20 survey, which includes a $32.2 \operatorname{arcmin}^{2}$ field within the CDF-S.

\subsection{Photometric Redshifts}

Several investigations for photometric redshifts in the CDF-S region have been made in the past. Particularly relevant is the study by the COMBO-17 project, which has observed this region with the Wide Field Imager (WFI) at the ESO $2.2 \mathrm{~m}$ telescope with a 17 band filter set covering the 350-930 nm wavelength range. As explained by Wolf et al. (2004), these "very low resolution spectra" allow accurate photometric redshifts to be obtained down to $R=23-24$ mag. Seeing-adaptive, weighted aperture 
TABLE 1

Identification of Faint Radio Sources

\begin{tabular}{|c|c|c|c|c|c|c|c|c|c|c|}
\hline $\begin{array}{l}\text { No. } \\
\text { (1) }\end{array}$ & $\begin{array}{l}\text { Radio Source } \\
\text { (2) }\end{array}$ & $\begin{array}{c}S_{1.4 \mathrm{GHz}} \\
(\mathrm{mJy}) \\
(3)\end{array}$ & $\begin{array}{l}\mathrm{ACS} \\
(4)\end{array}$ & $\begin{array}{l}\mathcal{R} \\
(5)\end{array}$ & $\begin{array}{c}\text { Distance } \\
\text { (arcsec) } \\
(6)\end{array}$ & $\begin{array}{l}z_{850} \\
(7)\end{array}$ & $\begin{array}{l}\text { CXO } \\
(8)\end{array}$ & $\begin{array}{c}\text { Distance } \\
(\operatorname{arcsec}) \\
(9)\end{array}$ & $\begin{array}{c}f_{0.5-2^{\mathrm{a}}} \\
(10)\end{array}$ & $\begin{array}{r}f_{2-8}{ }^{\mathrm{a}} \\
(11)\end{array}$ \\
\hline $1 \ldots \ldots \ldots$ & ATCDFS J033159.86-274541.3 & $0.137 \pm 0.042$ & & & $\cdots$ & $>27.7$ & & & & \\
\hline $2 \ldots \ldots \ldots$ & ATCDFS J033204.81-274125.8 & $0.095 \pm 0.038$ & J033204.84-274127.4 & 0.86 & 1.6 & 23.545 & J033204.89-274127.6 & 2.0 & $<0.23$ & 5.66 \\
\hline $3 \ldots \ldots \ldots$ & ATCDFS J033205.07-274535.5 & $0.087 \pm 0.042$ & J033205.21-274537.3 & 0.38 & 2.6 & 25.300 & & & & \\
\hline $4 \ldots \ldots \ldots$ & ATCDFS J033208.51-274648.8 & $0.193 \pm 0.040$ & J033208.53-274648.3 & 1.00 & 0.6 & 18.565 & J033208.50-274648.6 & 0.3 & $<0.09$ & 1.14 \\
\hline $5 \ldots \ldots \ldots$ & ATCDFS J033208.60-274043.0 & $0.077 \pm 0.038$ & J033208.67-274042.9 & 0.98 & 0.8 & 20.048 & & & & \\
\hline $6 \ldots \ldots \ldots$ & ATCDFS J033208.67-274734.3 & $1.958 \pm 0.046$ & J033208.66-274734.4 & 1.00 & 0.2 & 18.483 & J033208.66-274734.4 & 0.2 & 44.61 & 67.71 \\
\hline $7 \ldots \ldots \ldots$ & ATCDFS J033209.72-274249.0 & $0.229 \pm 0.039$ & J033209.71-274248.1 & 0.99 & 0.9 & 20.479 & J033209.69-274248.4 & 0.7 & 0.42 & $<0.62$ \\
\hline $8 \ldots \ldots \ldots$ & ATCDFS J033210.80-274629.2 & $0.157 \pm 0.042$ & J033210.79-274627.8 & 0.98 & 1.5 & 22.911 & $\mathrm{~J} 033210.80-274627.6$ & 1.7 & 0.09 & $<0.38$ \\
\hline $9 \ldots \ldots \ldots$. & ATCDFS J033210.91-274415.1 & $3.057 \pm 0.052$ & J033210.91-274414.9 & 1.00 & 0.2 & 22.371 & J033210.91-274415.1 & 0.1 & 6.14 & 9.81 \\
\hline $10 \ldots \ldots$ & ATCDFS J033211.00-274053.6 & $0.286 \pm 0.038$ & J033210.99-274053.7 & 0.99 & 0.2 & 23.577 & J033211.00-274053.7 & 0.1 & 1.88 & 3.94 \\
\hline $11 \ldots \ldots$. & ATCDFS J033211.51-274711.5 & $0.137 \pm 0.040$ & J033211.50-274713.1 & 0.99 & 1.5 & 21.086 & & & $\ldots$ & $\ldots$ \\
\hline $12 \ldots \ldots$. & ATCDFS J033213.08-274351.0 & $1.366 \pm 0.038$ & & & & $>28.2$ & & & & \\
\hline $13 a \ldots . .$. & ATCDFS J033213.28-274240.3 & $0.127 \pm 0.048$ & $\mathrm{~J} 033213.23-274241.0^{\mathrm{b}}$ & 0.91 & 0.9 & 19.698 & J033213.24-274240.9 & 0.8 & 2.48 & 18.73 \\
\hline $13 b \ldots . .$. & & & $\mathrm{J} 033213.37-274239.9^{\mathrm{b}}$ & & 1.3 & 21.919 & & & $\ldots$ & \\
\hline $14 \ldots \ldots$. & ATCDFS J033214.17-274910.2 & $0.141 \pm 0.034$ & J033214.13-274910.1 & 0.97 & 0.6 & 24.105 & & & & \\
\hline $15 \ldots \ldots$. & ATCDFS J033217.11-274303.9 & $0.079 \pm 0.040$ & J0332 & 0.98 & 0.7 & 20.566 & J033217.14-274303.3 & 0.7 & 4.50 & 6.39 \\
\hline $16 \ldots \ldots$. & ATCDFS J033217.22-275222.4 & $0.063 \pm 0.032$ & $\mathrm{~J} 033217.17-2$ & 0.70 & 1.8 & 21.766 & J033217.18-2 & 1.7 & 0.55 & 20.15 \\
\hline $17 \ldots \ldots$. & ATCDFS J033218.01-274718.4 & $0.404 \pm 0.034$ & J033218.01-274718.5 & 1.00 & 0.1 & 19.405 & $\mathrm{~J} 033218.07-274718.2$ & 0.7 & 0.28 & $<0.32$ \\
\hline $18 \ldots \ldots$. & ATCDFS J033218.03-275056.2 & $0.203 \pm 0.048$ & J033218.17-275056.6 & 0.43 & 1.8 & 26.263 & $\ldots$ & $\ldots$ & $\ldots$ & $\ldots$ \\
\hline $19 \ldots \ldots$. & ATCDFS J033218.62-275411.4 & $4.779 \pm 0.071$ & J033219.29-275406.1 & & 10.2 & 20.279 & $(\mathrm{G} 249)^{\mathrm{c}}$ & 12.4 & 0.15 & 0.61 \\
\hline $20 \ldots \ldots$ & ATCDFS J033219.41-275216.5 & $0.201 \pm 0.032$ & J033219.52-275217.7 & 0.55 & 1.8 & 21.871 & & $\ldots$ & $\ldots$ & $\ldots$ \\
\hline $21 \ldots \ldots$. & ATCDFS J033219.62-275402.9 & $5.861 \pm 0.083$ & J033219.29-275406.1 & $\ldots$ & 5.5 & 20.279 & $(\mathrm{G} 249)^{\mathrm{c}}$ & 4.0 & 0.15 & 0.61 \\
\hline $22 \ldots \ldots$. & ATCDFS J033219.82-274121.2 & $0.228 \pm 0.085$ & J033219.81-274122.7 & 0.98 & 1.5 & 18.736 & J033219.81-274123.1 & 1.9 & 0.22 & $<0.94$ \\
\hline $23 \ldots \ldots$ & ATCDFS J033221.24-274436.1 & $0.178 \pm 0.034$ & J033221.28-274435.6 & 0.97 & 0.7 & 19.625 & $\mathrm{~J} 033221.24-274435.9$ & 0.2 & $<0.08$ & $<0.31$ \\
\hline $24 \ldots \ldots$. & ATCDFS J033221.92-274243.8 & $0.089 \pm 0.040$ & J033222.01-274243.3 & 0.80 & 1.2 & 24.099 & $\ldots$ & $\ldots$ & $\ldots$ & $\ldots$ \\
\hline $25 \ldots \ldots$. & ATCDFS J033222.36-274807.3 & $0.144 \pm 0.042$ & & & $\ldots$ & $>28.4$ & $\ldots$ & $\ldots$ & $\ldots$ & $\ldots$ \\
\hline $26 \ldots \ldots$. & ATCDFS J0332 & $0.097 \pm 0.034$ & J033222.58-274425.8 & 0.97 & 1.5 & 20.279 & $\mathrm{~J} 033222.63-274426.0$ & 1.5 & $<0.07$ & $<0.43$ \\
\hline $27 \ldots \ldots$. & ATCDFS J033224.59-275441.6 & $0.086 \pm 0.038$ & J033224.53-2754 & 0.96 & 1.6 & 18.907 & & & $\ldots$ & $\ldots$ \\
\hline $28 \ldots \ldots$. & ATCDFS J033225.15-275452.2 & $0.079 \pm 0.038$ & J033225.16-275450.1 & 0.97 & 2.1 & 21.208 & J033225.17-275449.6 & 2.7 & $<0.08$ & 3.91 \\
\hline $29 \ldots \ldots$. & ATCDFS J033226.98-274106.5 & $20.011 \pm 0.208$ & J033227.01-274105.0 & 0.73 & 1.6 & 19.004 & J033227.00-274105.1 & 1.5 & 49.41 & 68.58 \\
\hline $30 \ldots \ldots$. & ATCDFS J033227.64-275040.5 & $0.076 \pm 0.032$ & J033227.72-275040.8 & 0.92 & 1.0 & 21.420 & $\mathrm{~J} 033227.67-275040.7$ & 0.3 & 0.03 & $<0.27$ \\
\hline $31 \ldots \ldots$. & ATCDFS J033227.98-274641.5 & $0.094 \pm 0.030$ & J033227.99-274639.2 & 0.96 & 2.3 & 19.364 & $\ldots$ & $\ldots$ & $\ldots$ & $\ldots$ \\
\hline $32 \ldots \ldots$. & ATCDFS J033228.31-273842.9 & $0.142 \pm 0.060$ & J033228.35-273841.7 & 0.99 & 1.4 & 19.246 & & $\ldots$ & $\ldots$ & $\ldots$ \\
\hline $33 \ldots \ldots$. & ATCDFS J033228.71-274402.3 & $4.061 \pm 0.083$ & & $\ldots$ & $\ldots$ & $>28.1$ & & $\ldots$ & $\ldots$ & $\ldots$ \\
\hline $34 \ldots \ldots$. & ATCDFS J033228.78-274620.8 & $0.237 \pm 0.036$ & J033228.74-274620.4 & 0.98 & 0.7 & 21.017 & $\mathrm{~J} 033228.73-274620.2$ & 0.9 & 0.25 & 0.20 \\
\hline $35 \ldots \ldots$. & ATCDFS J033229.59-274332.5 & $1.429 \pm 0.076$ & & & $\ldots$ & $>28.1$ & 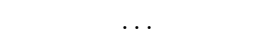 & $\ldots$ & $\ldots$ & $\ldots$ \\
\hline $36 \ldots \ldots$. & ATCDFS J033229.83-274423.7 & $1.088 \pm 0.044$ & J033229.88-274424.4 & 0.97 & 0.8 & 16.451 & J033229.88-27 & 1.3 & 0.81 & $<0.34$ \\
\hline $37 \ldots \ldots$. & ATCDFS J033229.89-274520.0 & $0.115 \pm 0.054$ & J033229.85-274520.5 & 0.91 & 0.7 & 21.009 & J033229.75-274520.3 & 1.9 & $<0.07$ & 0.13 \\
\hline $38 \ldots \ldots$. & ATCDFS J033229.96-274404.8 & $0.449 \pm 0.036$ & J033229.99-27 & 1.00 & 0.3 & 16.840 & $\mathrm{~J} 033230.01-274404.0$ & 1.0 & 0.57 & 0.71 \\
\hline $39 \ldots \ldots$. & ATCDFS J033229.98-275258.7 & $0.082 \pm 0.025$ & J033230.10-275300.1 & 0.40 & 2.0 & 26.190 & $\ldots$ & $\ldots$ & $\ldots$ & $\ldots$ \\
\hline $40 \ldots \ldots$ & ATCDFS J033230.20-275312.7 & $0.063 \pm 0.024$ & J033230.23-275312.4 & 0.62 & 0.5 & 26.759 & & . & $\ldots$ & \\
\hline $41 \mathrm{a} \ldots . .$. & ATCDFS J033231.44-274621.5 & $0.063 \pm 0.036$ & J033231.46-274623.2 & 0.49 & 1.6 & 22.877 & J033231.47-274623.0 & 1.4 & 0.06 & $<0.33$ \\
\hline $41 \mathrm{~b} \ldots . .$. & & & J033231.40-274621.4 & 0.48 & 0.6 & 23.046 & $\ldots$ & $\ldots$ & $\ldots$ & $\ldots$ \\
\hline $42 \ldots \ldots$ & ATCDFS J033233.44-275228.0 & $0.097 \pm 0.032$ & & & $\ldots$ & $>28.1$ & $(\mathrm{G} 632)^{\mathrm{c}}$ & 1.5 & $<0.06$ & 0.46 \\
\hline $43 \ldots \ldots$. & ATCDFS J033234.97-275456.1 & $0.127 \pm 0.030$ & $\mathrm{~J} 033234.96-275455.8^{\mathrm{d}}$ & 0.98 & 0.4 & 27.482 & $\ldots$ & $\ldots$ & $\ldots$ & $\ldots$ \\
\hline $44 \ldots \ldots$. & ATCDFS J033235.07-275532.8 & $0.310 \pm 0.048$ & J033235.08-275533.0 & 1.00 & 0.2 & 15.698 & J033234.73-275533.8 & 4.6 & $<0.13$ & 1.66 \\
\hline $45 \ldots \ldots$. & ATCDFS J033235.46-275452.8 & $0.078 \pm 0.028$ & J033235.51-275449.9 & 0.56 & 3. & 26.166 & & $\ldots$ & $\ldots$ & $\ldots$ \\
\hline $46 \ldots \ldots$. & ATCDFS J033235.71-274916.0 & $0.072 \pm 0.030$ & J033235.71-274916.0 & 0.97 & 0 . & 24.660 & J033235.72-274916.0 & 0.1 & 0.05 & 0.70 \\
\hline $47 \mathrm{a} \ldots .$. & ATCDFS J033237.22-275129.7 & $0.077 \pm 0.032$ & J033237.17-275127.9 & 0.90 & 1.9 & 21.352 & $\ldots$ & $\ldots$ & $\ldots$ & \\
\hline $47 \mathrm{~b} \ldots .$. & & & J033237.34-275127.4 & & 2.8 & 21.028 & $\cdots$ & $\ldots$ & $\ldots$ & \\
\hline $48 \mathrm{a} \ldots .$. & ATCDFS J033237.75-275000.7 & $0.181 \pm 0.038$ & J033237.74-275000.4 & 0.51 & 0.4 & 23.353 & $\ldots$ & $\ldots$ & $\ldots$ & $\ldots$ \\
\hline $48 \mathrm{~b} \ldots .$. & & & J033237.76-275001.4 & 0.48 & 0.6 & 23.310 & & & & \\
\hline $49 \ldots \ldots$ & ATCDFS J033237.75-275211.9 & $0.091 \pm 0.032$ & J033237.76-275212.3 & 0.98 & 0.3 & 23.540 & J033237.77-275212.4 & 0.5 & 5.97 & 8.78 \\
\hline $50 \ldots \ldots$ & ATCDFS J033238.75-274632.5 & $0.072 \pm 0.030$ & J033238.60-274631.4 & 0.23 & 2.4 & 21.533 & $\ldots$ & $\ldots$ & $\ldots$ & $\ldots$ \\
\hline $51 \mathrm{a} \ldots . .$. & ATCDFS J033239.46-275300.9 & $0.127 \pm 0.032$ & J033239.47-275300.5 & 0.57 & 0.5 & 20.540 & & $\ldots$ & & \\
\hline $51 \mathrm{~b} \ldots . .$. & & & J033239.49-275301.6 & 0.43 & 0.7 & 20.400 & $\ldots$ & $\ldots$ & $\ldots$ & $\ldots$ \\
\hline $52 \mathrm{a} \ldots . .$. & ATCDFS J033239.64-274851.8 & $0.066 \pm 0.030$ & J033239.67-274850.6 & 0.49 & 1.3 & 24.547 & J033239.68-274850.7 & 1.2 & 0.75 & 7.06 \\
\hline $52 \mathrm{~b} \ldots . .$. & & & J033239.56-274851.7 & 0.47 & 1.2 & 22.552 & 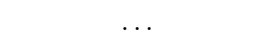 & $\ldots$ & $\ldots$ & $\ldots$ \\
\hline $53 \ldots \ldots$. & ATCDFS J033240.82-275545.8 & $0.102 \pm 0.032$ & J033240.84-275546.7 & 0.97 & & 25.183 & J033240.84-275546.6 & 0.8 & 0.54 & 9.34 \\
\hline $54 \ldots \ldots$. & ATCDFS J033243.12-275514.2 & $0.070 \pm 0.034$ & J033243.17-275514.7 & 0.79 & 0.7 & 23.991 & $\ldots$ & $\ldots$ & $\ldots$ & \\
\hline $55 \mathrm{a} \ldots .$. & ATCDFS J033244.16-275142.4 & $0.511 \pm 0.030$ & J033244.05-275143.3 & $\ldots$ & 1.7 & 18.491 & & $\cdots$ & $\cdots$ & \\
\hline $55 \mathrm{~b} \ldots .$. & & & J033244.27-275141.1 & & 1.9 & 19.166 & $\mathrm{~J} 033244.28-275141.0$ & 2.1 & 0.51 & $<0.33$ \\
\hline $56 \ldots \ldots$. & ATCDFS J033244.93-274726.2 & $0.107 \pm 0.033$ & J033244.86-274727.6 & 0.93 & 1.6 & 18.456 & 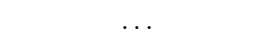 & .. & $\ldots$ & $\ldots$ \\
\hline
\end{tabular}


TABLE 1-Continued

\begin{tabular}{|c|c|c|c|c|c|c|c|c|c|c|}
\hline $\begin{array}{l}\text { No. } \\
\text { (1) }\end{array}$ & $\begin{array}{l}\text { Radio Source } \\
\text { (2) }\end{array}$ & $\begin{array}{c}S_{1.4 \mathrm{GHz}} \\
(\mathrm{mJy}) \\
(3)\end{array}$ & $\begin{array}{l}\text { ACS } \\
(4)\end{array}$ & $\begin{array}{l}\mathcal{R} \\
(5)\end{array}$ & $\begin{array}{c}\text { Distance } \\
(\operatorname{arcsec}) \\
(6)\end{array}$ & $\begin{array}{l}z_{850} \\
(7)\end{array}$ & $\begin{array}{l}\mathrm{CXO} \\
(8)\end{array}$ & $\begin{array}{c}\text { Distance } \\
(\operatorname{arcsec}) \\
(9)\end{array}$ & $\begin{array}{c}f_{0.5-2^{\mathrm{a}}} \\
(10)\end{array}$ & $\begin{array}{l}f_{2-8}{ }^{\mathrm{a}} \\
(11)\end{array}$ \\
\hline $57 \ldots \ldots .$. & ATCDFS J033245.03-275438.8 & $0.122 \pm 0.038$ & J033245.02-275439.6 & 0.99 & 0.7 & 18.987 & J033244.98-275438.7 & 0.7 & 0.14 & $<0.60$ \\
\hline $58 \ldots \ldots .$. & ATCDFS J033246.03-275318.2 & $0.219 \pm 0.046$ & $\ldots$ & $\ldots$ & $\ldots$ & $>28.2$ & $\ldots$ & $\ldots$ & $\ldots$ & $\ldots$ \\
\hline $59 \ldots \ldots \ldots$ & ATCDFS J033246.33-275328.6 & $0.080 \pm 0.038$ & J033246.33-275327.0 & 0.76 & 1.7 & 23.639 & $\ldots$ & $\ldots$ & $\ldots$ & $\ldots$ \\
\hline $60 \mathrm{a} \ldots . .$. & ATCDFS J033246.78-275120.1 & $0.079 \pm 0.030$ & J033246.84-275121.2 & 0.51 & 1.3 & 23.562 & $\ldots$ & $\ldots$ & $\ldots$ & $\ldots$ \\
\hline $60 \mathrm{~b} \ldots \ldots$ & & & J033246.79-275118.8 & 0.45 & 1.4 & 24.769 & $\ldots$ & $\ldots$ & $\ldots$ & $\ldots$ \\
\hline $61 \ldots \ldots .$. & ATCDFS J033248.02-275414.7 & $0.103 \pm 0.047$ & J033248.05-275412.4 & 0.59 & 2.4 & 26.566 & $\ldots$ & $\ldots$ & $\ldots$ & $\ldots$ \\
\hline $62 \ldots \ldots .$. & ATCDFS J033248.62-274934.9 & $0.097 \pm 0.030$ & J033248.57-274934.3 & 0.92 & 1.0 & 23.425 & $(\mathrm{G} 578)^{\mathrm{c}}$ & 0.5 & 0.09 & $<0.36$ \\
\hline $63 \ldots \ldots \ldots$ & ATCDFS J033300.91-275049.4 & $0.078 \pm 0.036$ & J033300.98-275053.7 & 0.60 & 4.3 & 21.675 & $\ldots$ & $\ldots$ & $\ldots$ & $\ldots$ \\
\hline $64 \ldots \ldots \ldots$ & ATCDFS J033302.99-275147.3 & $0.080 \pm 0.036$ & $\mathrm{~J} 033303.05-275145.8$ & 0.89 & 1.7 & 25.134 & J033302.97-275146.4 & 1.0 & 0.69 & 0.93 \\
\hline
\end{tabular}

Note.-Table 1 is also available in machine-readable form in the electronic version of the Astronomical Journal.

${ }^{\mathrm{a}}$ Units of $10^{-18} \mathrm{~W} \mathrm{~m}^{-2}$.

${ }^{\mathrm{b}}$ Optical sources deblended using SExtractor.

${ }^{c}$ X-ray sources from Giacconi et al. (2002). The source is denoted by its number in the catalog therein, while the flux in the $2-8 \mathrm{keV}$ band is an estimate based on the reported flux in the $2-10 \mathrm{keV}$ band and assuming a power-law photon index of 1.4 .

${ }^{\mathrm{d}}$ Optical source not in the released ACS catalog. The $z_{850}$ magnitude results from aperture photometry.

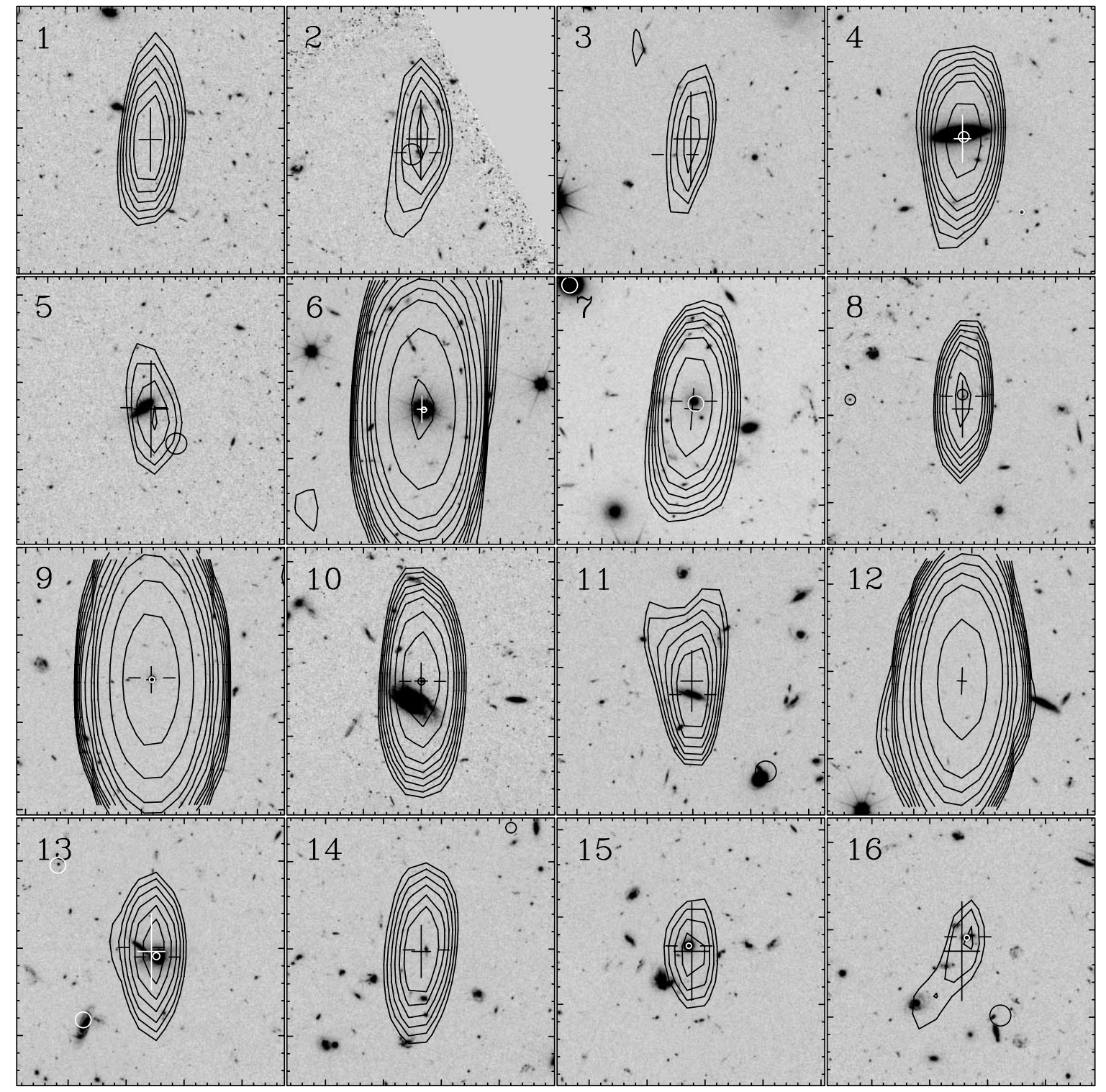

FIG. 2.-Optical $B_{435}+V_{606}+i_{775}+z_{850}$ images for radio sources in the GOODS CDF-S ACS field with radio contours overlaid and X-ray detections indicated. The source number from Table 1 is printed in the upper left corner of each image, which is roughly $30^{\prime \prime}$ wide, centered on the radio source coordinates and with north being up and east to the right. The crosses indicate the $3 \sigma$ radio position error regions. Radio contours go from 45 to $93 \mu \mathrm{Jy}$ in factors of 1.2 , from 110 to $302 \mu \mathrm{Jy}$ in factors of 1.4 , and then from $420 \mu \mathrm{Jy}$ to $13 \mathrm{mJy}$ in factors of 2 . The horizontal lines enclose the assumed optical counterparts of radio sources, while circles indicate X-ray detections with sizes that denote the X-ray position error. For sources 19, 21, 42, and 62, the circles indicate X-ray detections present only in the Giacconi et al. (2002) catalog. 


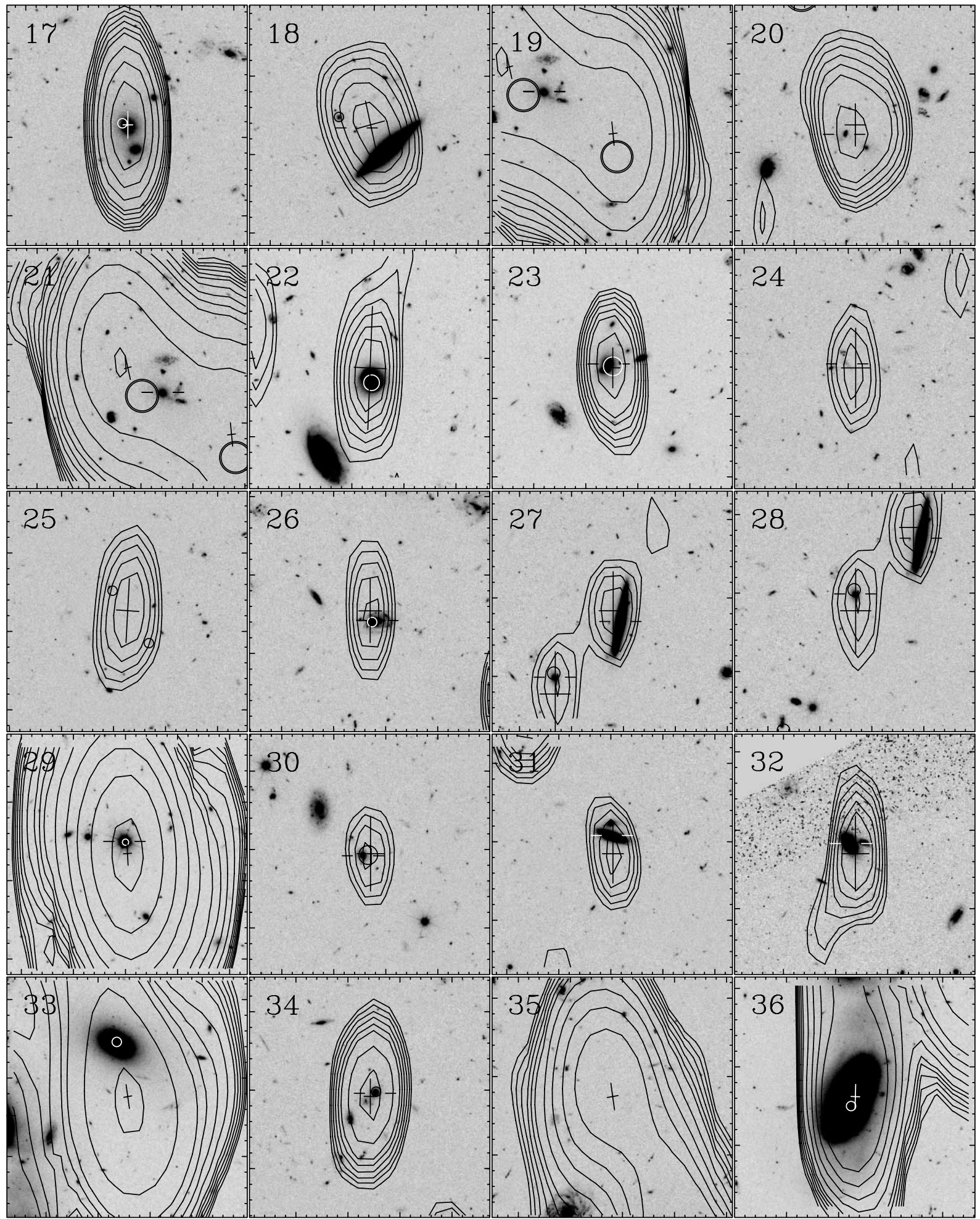

FIG. 2.-Continued

photometry is used to measure the same fraction of an object in every band independently of seeing effects; for most objects this is similar to a flux measurement in an aperture of 1 ."5 diameter in 1.5 seeing (Wolf et al. 2004). The spectral energy distribution templates used include star, galaxy (with and without extinction), and QSO types. Mean redshift and variance $(1 \sigma)$, as well as the classification from the best-fit procedure, were extracted from the catalog (Wolf et al. 2004) and are presented here.
For optically fainter sources, eventually at higher redshifts, the near-infrared bands not covered by the COMBO-17 data set assume a more important role. To get a photometric redshift estimate for these objects, we have also searched the catalog of Mobasher et al. (2004), which uses ground-based optical ( $U^{\prime} U B V R I$ bands) and near-infrared $\left(J H K_{s}\right)$ data from ESO facilities (WFI at the $2.2 \mathrm{~m}$ telescope, FORS 1 and ISAAC at the VLT, and SOFI at the New Technology Telescope), as well as 


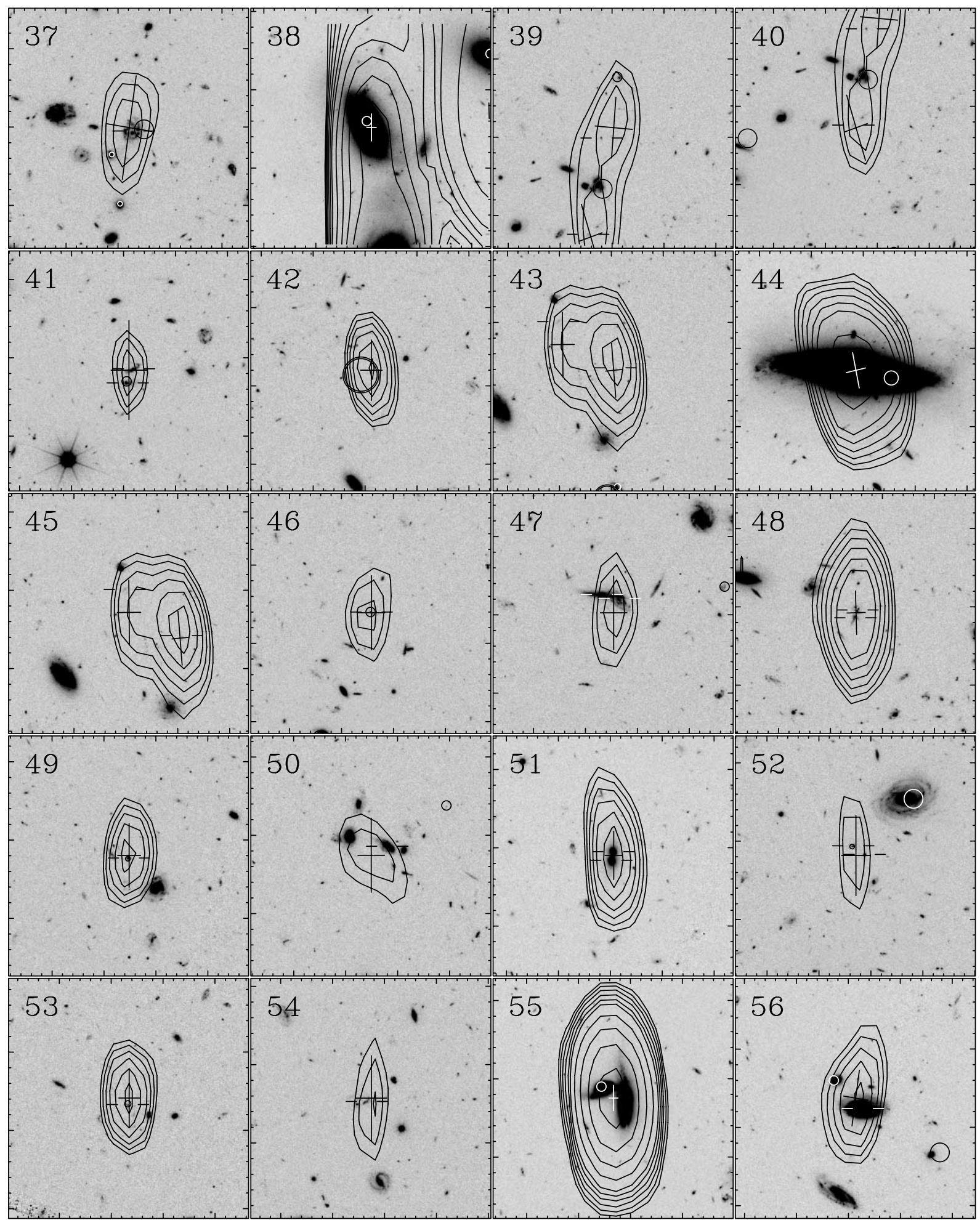

Fig. 2.-Continued

space observations from the $H S T \operatorname{ACS}\left(B_{435}, V_{606}, i_{775}\right.$, and $\left.z_{850}\right)$, resulting in as many as 18 independent photometric measurements for each source. While useful for fainter sources, the spectral libraries used do not include QSO templates, which results in a lack of accuracy for some sources, as can be seen in this paper. Also, the photometry measurements in Mobasher et al. (2004) were made through matched $3^{\prime \prime}$ diameter apertures in all bands. In the case of some optical sources with very close neighbors, often only identified in the ACS images, this can bias the photometric redshift estimate; we note such cases when commenting on the individual sources.

\subsection{X-Ray and Radio Luminosities}

Luminosities were estimated whenever a redshift determination existed. In order of reliability we have considered the spectroscopic redshift or, in its absence, the photometric redshift from the 


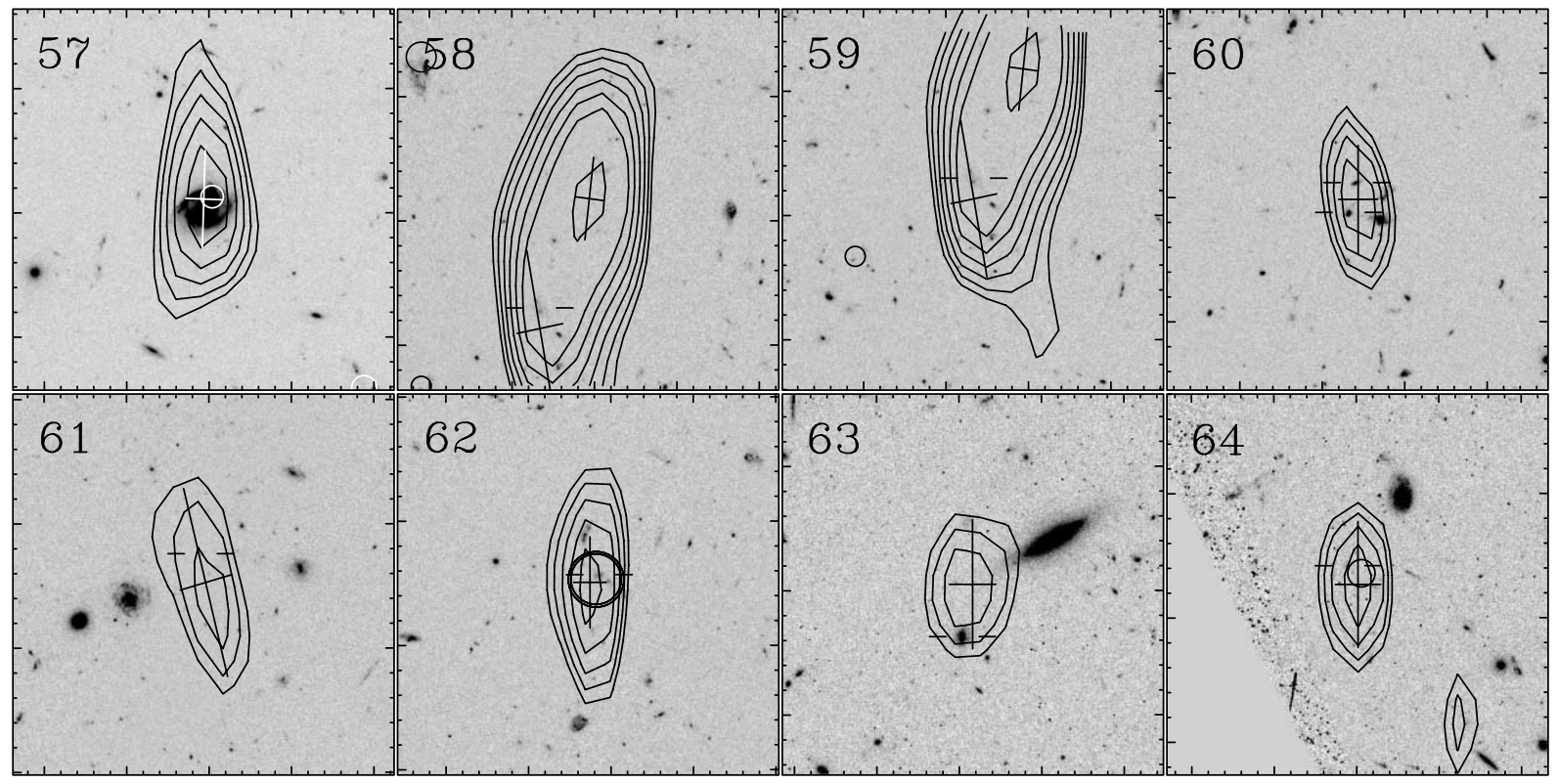

FIG. 2.-Continued

COMBO-17 work. For faint sources with no other redshift information, the estimate from Mobasher et al. (2004) was used.

The rest-frame X-ray luminosity was calculated as

$$
L_{\mathrm{X}}=4 \pi d_{L}^{2} f_{\mathrm{X}}(1+z)^{\Gamma-2} \operatorname{ergs~s}^{-1},
$$

where $d_{L}$ is the luminosity distance $(\mathrm{cm}), f_{\mathrm{X}}$ is the $\mathrm{X}$-ray flux in the $0.5-8 \mathrm{keV}$ band (ergs cm${ }^{-2} \mathrm{~s}^{-1}$ ), and $\Gamma$ is the photon index, taken from Alexander et al. (2003). For sources detected only in the Giacconi et al. (2002) catalogs, the $0.5-8 \mathrm{keV}$ X-ray flux is taken as the sum of the $0.5-2$ and the $2-8 \mathrm{keV}$ fluxes, where the latter is derived from the quoted $2-10 \mathrm{keV}$ flux assuming $\Gamma=1.4$, as explained above.

The rest-frame radio luminosity density was calculated as

$$
L_{1.4 \mathrm{GHz}}=4 \pi d_{L}^{2} S_{1.4 \mathrm{GHz}} 10^{-33}(1+z)^{\alpha-1} \mathrm{~W} \mathrm{~Hz}^{-1},
$$

where $d_{L}$ is the luminosity distance $(\mathrm{cm}), S_{1.4 \mathrm{GHz}}$ is the $1.4 \mathrm{GHz}$ flux density (millijanskys), and $\alpha$ is the radio spectral index $\left(f_{\nu} \propto \nu^{-\alpha}\right)$. In determining the radio luminosity density, we have assumed $\alpha=0.8$, the characteristic radio spectral index of synchrotron radiation. Of the 65 optical sources considered in Table 1, 34 have a spectroscopic redshift, while a further 24 have a photometric redshift estimate.

\subsection{Classification}

We adopt a classification based primarily on the observed $\mathrm{X}$-ray properties, $L_{\mathrm{X}}$ and hardness ratio (HR). We derive $L_{\mathrm{X}}$ from the $0.5-8 \mathrm{keV}$ fluxes and spectroscopic or photometric redshifts, while $\mathrm{HR}$ is calculated as $\mathrm{HR}=(H-S) /(H+S)$, with $H$ and $S$ being the count rates in the hard $(2-8 \mathrm{keV})$ and soft $(0.5-2 \mathrm{keV})$ bands, respectively, taken from Alexander et al. (2003). For sources detected in X-rays only in the Giacconi et al. (2002) catalogs, we assume the quoted HR derived from the $0.5-2$ and 2$10 \mathrm{keV}$ band counts, neglecting the (small) discrepancy arising from the different hard band used. Our classification rests on the criteria adopted by Szokoly et al. (2004), namely,

Galaxy.-With $L_{\mathrm{X}}<10^{42} \mathrm{ergs} \mathrm{s}^{-1}$ and $\mathrm{HR} \leq-0.2$.
$A G N-2$.-With $\quad 10^{41} \mathrm{ergs} \mathrm{s}^{-1} \leq L_{\mathrm{X}}<10^{44} \mathrm{ergs} \mathrm{s}^{-1} \quad$ and $\mathrm{HR}>-0.2$.

$A G N-1$.-With $10^{42} \mathrm{ergs} \mathrm{s}^{-1} \leq L_{\mathrm{X}}<10^{44} \mathrm{ergs} \mathrm{s}^{-1} \quad$ and $\mathrm{HR} \leq-0.2$.

QSO-2.-With $L_{\mathrm{X}} \geq 10^{44} \mathrm{ergs} \mathrm{s}^{-1}$ and HR $>-0.2$.

$Q S O-1$. - With $L_{\mathrm{X}} \geq 10^{44} \mathrm{ergs} \mathrm{s}^{-1}$ and $\mathrm{HR} \leq-0.2$.

In view of the different $X$-ray catalogs employed here, the different bands used to calculate $L_{\mathrm{X}}(0.5-8 \mathrm{keV}$ instead of $0.5-$ $10 \mathrm{keV}$ ), and the different $K$-corrections employed in the calculation of $L_{\mathrm{X}}$, we note that there are some (expected) differences between the X-ray classification presented in Szokoly et al. (2004) and the one herein for some galaxies that appear in both works.

Among sources with insufficient X-ray information (or no $\mathrm{X}$-ray detection), an AGN identification was assigned whenever $L_{\mathrm{X}} \geq 10^{42} \mathrm{ergs} \mathrm{s}^{-1}$ (source classified as AGNX) or $L_{1.4 \mathrm{GHz}} \geq$ $10^{24.5} \mathrm{~W} \mathrm{~Hz}^{-1}$ (source classified as AGNR), as derived from the spectroscopic redshift or the most likely photometric redshift estimate. The adopted radio luminosity limit is derived from the radio luminosity distribution for star-forming galaxies and AGNs observed in the Phoenix Deep Survey (Afonso et al. 2005).

In addition, the optical spectroscopic information, when available, was used. Since most sources with a spectrum were observed by Szokoly et al. (2004), we adopted their optical classifications: BLAGN for whenever the emission lines are broad (FWHM $>2000 \mathrm{~km} \mathrm{~s}^{-1}$ ); HEX for galaxies displaying narrow emission lines, some of which are not found in $\mathrm{H}$ in regions (e.g., [Ne v] 23425, He II 21640 ); LEX for objects with an $\mathrm{H}$ II region-type spectrum; and ABS for galaxies with an absorption-line spectrum. Spectra from Le Fèvre et al. (2004), Vanzella et al. (2005), and Mignoli et al. (2005) were visually inspected and classified accordingly. Finally, diagnostic emission-line ratios were used to separate Seyfert 2 galaxies from star-forming galaxies whenever suitable emission lines of significant $\mathrm{S} / \mathrm{N}$ were present, providing an extra degree of information from the optical spectra.

The final characterization of the radio sample is presented in Table 2, which contains the following information: Column (1) is the source number. Column (2) is the spectroscopic redshift. Column (3) is the origin of the spectroscopic redshift. Column (4) 
TABLE 2

Characterization of Faint Radio Sources

\begin{tabular}{|c|c|c|c|c|c|c|c|c|c|c|c|c|c|c|c|c|}
\hline \multirow[b]{2}{*}{$\begin{array}{l}\text { No. } \\
(1)\end{array}$} & \multirow[b]{2}{*}{$\begin{array}{l}z_{\mathrm{sp}} \\
(2)\end{array}$} & \multirow[b]{2}{*}{$\begin{array}{c}\text { ORIGIN }^{\mathrm{a}} \\
\text { (3) }\end{array}$} & \multirow[b]{2}{*}{$\begin{array}{l}Q_{z} \\
(4)\end{array}$} & \multicolumn{2}{|c|}{ COMBO-17 } & \multicolumn{3}{|c|}{ MoBASHER ET AL. (2004) } & \multirow[b]{2}{*}{$\begin{array}{c}L_{1.4 \mathrm{GHz}} \\
\left(\mathrm{W} \mathrm{Hz}^{-1}\right) \\
(10)\end{array}$} & \multirow[b]{2}{*}{$\begin{array}{c}L_{\mathrm{X}} \\
\left(\mathrm{ergs} \mathrm{s}^{-1}\right) \\
(11)\end{array}$} & \multirow[b]{2}{*}{$\begin{array}{c}f_{1.4} / f_{\text {opt }} \\
\quad(12)\end{array}$} & \multirow[b]{2}{*}{$\begin{array}{c}f_{\mathrm{X}} / f_{\mathrm{opt}} \\
\quad(13)\end{array}$} & \multirow[b]{2}{*}{$\begin{array}{l}\text { HR } \\
(14)\end{array}$} & \multirow[b]{2}{*}{$\begin{array}{c}\Gamma \\
(15)\end{array}$} & \multirow[b]{2}{*}{$\begin{array}{c}\text { Class (X-RAYS } \\
\text { or Radio) } \\
(16)\end{array}$} & \multirow[b]{2}{*}{$\begin{array}{c}\text { Class } \\
\text { (Optical) } \\
(17)\end{array}$} \\
\hline & & & & $\begin{array}{l}z_{\mathrm{ph}} \\
(5)\end{array}$ & $\begin{array}{c}\text { Type } \\
\text { (6) }\end{array}$ & $\begin{array}{l}z_{\mathrm{ph}} \\
(7)\end{array}$ & $\begin{array}{c}\text { Type } \\
(8)\end{array}$ & $\begin{array}{c}\text { No. of } \\
\text { Bands } \\
\text { (9) }\end{array}$ & & & & & & & & \\
\hline 1 1.................................. & & $\ldots$ & $\ldots$ & & $\ldots$ & $\ldots$ & $\ldots$ & $\ldots$ & $\ldots$ & $\ldots$ & $>3.66$ & $\ldots$ & & $\ldots$ & & $\ldots$ \\
\hline 2 & 0.720 & $\mathrm{~S}$ & 1.0 & $0.686 \pm 0.080$ & Galaxy & $1.29(0.99-1.59)$ & 2.67 & 13 & 23.30 & 42.49 & 1.84 & $<-0.10$ & $>0.51$ & $<-0.27$ & AGN-2 & LEX \\
\hline $3 \ldots \ldots \ldots \ldots \ldots \ldots \ldots \ldots \ldots$ & & $\ldots$ & $\ldots$ & $\ldots$ & $\ldots$ & $0.43(0.13-3.73)$ & 2.00 & 5 & 22.73 & $\ldots$ & 2.50 & $\ldots$ & & $\ldots$ & & $\ldots$ \\
\hline 4 & 0.310 & $\mathrm{~S}$ & 2.0 & $0.328 \pm 0.004$ & Galaxy & $0.31(0.14-0.48)$ & 1.00 & 15 & 22.76 & 41.34 & 0.15 & $<-2.50$ & $>0.29$ & $<0.21$ & AGN-2 & LEX/Sey2 \\
\hline $5 \ldots \ldots \ldots \ldots \ldots \ldots \ldots \ldots \ldots$ & & $\ldots$ & $\ldots$ & $0.347 \pm 0.026$ & Galaxy & $0.44(0.25-0.63)$ & 2.00 & 13 & 22.47 & $\ldots$ & 0.35 & $\ldots$ & $\ldots$ & $\ldots$ & $\ldots$ & $\ldots$ \\
\hline 6 & 0.543 & $\mathrm{~S}$ & 2.0 & $0.582 \pm 0.017$ & QSO & $0.26(0.09-0.56)$ & 3.67 & 15 & 24.32 & 44.08 & 1.13 & 0.16 & -0.50 & 1.73 & QSO-1 & BLAGN \\
\hline 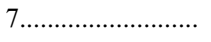 & 0.733 & $\mathrm{~S}$ & 2.0 & $0.762 \pm 0.007$ & Galaxy & $0.71(0.49-0.93)$ & 1.00 & 13 & 23.70 & 42.28 & 0.99 & -1.07 & $<-0.52$ & $>1.75$ & AGN-1 & LEX \\
\hline 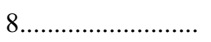 & 1.610 & M & 2.0 & $1.013 \pm 0.212$ & Galaxy & $1.32(1.02-1.62)$ & 1.00 & 14 & 24.34 & 42.47 & 1.80 & -0.76 & $<-0.05$ & $1.40^{\mathrm{b}}$ & AGNX & LEX \\
\hline 9 & 1.615 & $\mathrm{~S}$ & 2.0 & $1.595 \pm 0.010$ & QSO & $0.76(0.53-1.10)$ & 3.67 & 18 & 25.63 & 44.32 & 2.87 & 0.86 & -0.49 & 1.69 & QSO-1 & BLAGN \\
\hline $10 \ldots \ldots \ldots \ldots \ldots \ldots$ & & & & & & & & & & 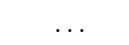 & 2.33 & 0.82 & -0.40 & 1.50 & Uncl. & \\
\hline 11 & 0.576 & $\mathrm{M}$ & 2.0 & $0.594 \pm 0.019$ & Galaxy & $0.94(0.69-1.19)$ & 2.00 & 18 & 23.23 & $\ldots$ & 1.01 & $\ldots$ & $\ldots$ & $\ldots$ & $\ldots$ & LEX \\
\hline $12 \ldots \ldots \ldots$ & $\ldots$ & $\ldots$ & $\ldots$ & $\ldots$ & $\ldots$ & $\ldots$ & $\ldots$ & $\ldots$ & $\ldots$ & $\ldots$ & $>4.86$ & $\ldots$ & $\ldots$ & $\ldots$ & $\ldots$ & $\ldots$ \\
\hline 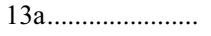 & 0.605 & $\mathrm{~S}$ & 2.0 & $0.609 \pm 0.008$ & Galaxy & $0.63(0.42-0.84)$ & 2.33 & 14 & 23.24 & 43.21 & 0.42 & -0.61 & 0.09 & 0.58 & AGN-2 & HEX \\
\hline $13 \mathrm{~b} \ldots \ldots \ldots \ldots \ldots \ldots \ldots$ & $\ldots$ & $\ldots$ & $\ldots$ & $\ldots$ & $\ldots$ & $\ldots$ & $\ldots$ & $\ldots$ & $\ldots$ & $\ldots$ & 1.31 & $\ldots$ & $\ldots$ & $\ldots$ & $\ldots$ & $\ldots$ \\
\hline $14 \ldots \ldots \ldots \ldots \ldots \ldots$ & $\ldots$ & $\ldots$ & $\ldots$ & $\ldots$ & $\ldots$ & $1.66(1.31-2.26)$ & 4.00 & 15 & 24.33 & $\ldots$ & 2.23 & $\ldots$ & $\ldots$ & $\ldots$ & $\ldots$ & $\ldots$ \\
\hline $15 \ldots \ldots$ & 0.569 & $\mathrm{~S}$ & 2.0 & $0.583 \pm 0.018$ & Galaxy & $0.59(0.38-0.80)$ & 2.00 & 15 & 22.98 & 43.12 & 0.56 & 0.00 & -0.52 & 1.77 & AGN-1 & BLAGN \\
\hline $16 \ldots$ & 1.097 & $\mathrm{~S}$ & 2.0 & $0.843 \pm 0.047$ & Galaxy & $1.09(0.82-1.36)$ & 1.67 & 14 & 23.55 & 43.28 & 0.95 & -0.43 & 0.63 & -0.58 & AGN-2 & LEX \\
\hline 17 & 0.734 & $\mathrm{~S}$ & 2.0 & $0.759 \pm 0.012$ & Galaxy & $0.77(0.54-1.00)$ & 1.00 & 17 & 23.95 & 41.96 & 0.81 & -1.67 & $<-0.58$ & $>1.92$ & Gal. & LEX \\
\hline 18 & $\ldots$ & $\ldots$ & $\ldots$ & $\ldots$ & $\ldots$ & $1.04(0.77-1.46)$ & 3.67 & 17 & 24.01 & $\ldots$ & 3.25 & $\ldots$ & $\ldots$ & $\ldots$ & $\ldots$ & $\ldots$ \\
\hline 19 & $\ldots$ & $\ldots$ & $\ldots$ & $0.965 \pm 0.014$ & Galaxy & $0.92(0.67-1.17)$ & 1.00 & 14 & 25.30 & 42.38 & 2.23 & -1.59 & $(-0.12)^{c}$ & $\ldots$ & AGN-2 & $\ldots$ \\
\hline 20 & $\ldots$ & $\ldots$ & $\ldots$ & $1.049 \pm 0.150$ & Galaxy & $1.07(0.80-1.34)$ & 1.33 & 13 & 24.01 & $\ldots$ & 1.49 & $\ldots$ & & $\ldots$ & & $\ldots$ \\
\hline $21 \ldots \ldots \ldots \ldots$ & & & & $0.965 \pm 0.014$ & Galaxy & $0.92(0.67-1.17)$ & 1.00 & 14 & 25.39 & 42.38 & 2.32 & -1.59 & $(-0.12)^{\mathrm{c}}$ & & AGN-2 & \\
\hline $22 \ldots \ldots \ldots \ldots \ldots \ldots$ & 0.229 & $\mathrm{~S}$ & 2.0 & $0.226 \pm 0.013$ & Galaxy & $0.24(0.08-0.40)$ & 1.33 & 14 & 22.53 & 41.21 & 0.29 & -2.04 & $<-0.15$ & $>1.00$ & Uncl. & LEX/Sey2 \\
\hline $23 \ldots \ldots \ldots \ldots$ & 0.524 & M & 2.0 & $0.460 \pm 0.018$ & Galaxy & $0.54(0.34-0.74)$ & 1.00 & 18 & 23.25 & 41.13 & 0.54 & $<-2.13$ & $\ldots$ & $\ldots$ & Uncl. & LEX \\
\hline $24 \ldots \ldots \ldots \ldots \ldots$ & $\ldots$ & $\ldots$ & $\ldots$ & $\ldots$ & $\ldots$ & $1.54(1.21-1.87)$ & 2.00 & 12 & 24.05 & $\ldots$ & 2.03 & $\ldots$ & $\ldots$ & $\ldots$ & $\ldots$ & $\ldots$ \\
\hline $25 \ldots \ldots \ldots \ldots \ldots \ldots$ & $\ldots$ & $\ldots$ & $\ldots$ & $\ldots$ & $\ldots$ & $\ldots$ & $\ldots$ & $\ldots$ & $\ldots$ & $\ldots$ & $>3.96$ & $\ldots$ & $\ldots$ & $\ldots$ & $\ldots$ & $\ldots$ \\
\hline $26 \ldots \ldots \ldots \ldots \ldots$ & 0.737 & $\mathrm{~S}$ & 2.0 & $0.736 \pm 0.015$ & Galaxy & $0.74(0.51-0.97)$ & 2.00 & 18 & 23.33 & 41.72 & 0.54 & $<-1.92$ & $\ldots$ & $1.40^{\mathrm{b}}$ & Uncl. & LEX/SF \\
\hline $27 \ldots \ldots \ldots \ldots \ldots \ldots$ & 0.123 & $\mathrm{~L}$ & 2.0 & $0.120 \pm 0.010$ & Galaxy & $0.18(0.03-0.33)$ & 2.00 & 15 & 21.52 & $\ldots$ & -0.06 & $\ldots$ & $\ldots$ & $\ldots$ & & LEX \\
\hline $28 \ldots \ldots \ldots \ldots \ldots$ & 1.090 & $\mathrm{~S}$ & 2.0 & $1.143 \pm 0.065$ & Galaxy & $1.01(0.75-1.27)$ & 1.33 & 14 & 23.64 & 42.46 & 0.82 & $<-1.49$ & $>0.69$ & $<-0.76$ & AGN-2 & ABS \\
\hline $29 \ldots \ldots \ldots \ldots \ldots$ & 0.734 & $\mathrm{~S}$ & 2.0 & $0.754 \pm 0.155$ & QSO & $0.07(0.00-0.54)$ & 6.00 & 14 & 25.64 & 44.42 & 2.34 & 0.42 & -0.53 & 1.79 & QSO-1 & BLAGN \\
\hline $30 \ldots \ldots \ldots \ldots \ldots \ldots \ldots$ & 1.097 & $\mathrm{~V}$ & 2.0 & $1.119 \pm 0.039$ & Galaxy & $1.06(0.79-1.33)$ & 2.00 & 18 & 23.63 & 41.58 & 0.89 & -1.83 & $<0.33$ & $\ldots$ & Uncl. & HEX \\
\hline 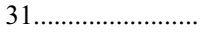 & 0.247 & $\mathrm{C}$ & 2.0 & $0.240 \pm 0.030$ & Galaxy & $0.47(0.28-0.66)$ & 1.33 & 18 & 22.22 & $\ldots$ & 0.16 & $\ldots$ & $\ldots$ & $\ldots$ & $\ldots$ & LEX \\
\hline 32 & .. & $\ldots$ & $\ldots$ & $0.391 \pm 0.012$ & Galaxy & $0.45(0.26-0.64)$ & 1.33 & 9 & 22.85 & $\ldots$ & 0.29 & $\ldots$ & $\ldots$ & $\ldots$ & $\ldots$ & $\ldots$ \\
\hline 33...................... & & & & & & & & & & & $>5.29$ & $\ldots$ & & $\ldots$ & & $\ldots$ \\
\hline $34 \ldots \ldots \ldots \ldots \ldots \ldots$ & 0.738 & $\mathrm{~S}$ & 2.0 & $0.710 \pm 0.030$ & Galaxy & $0.76(0.53-0.99)$ & 1.33 & 17 & 23.72 & 42.08 & 1.22 & -1.08 & -0.67 & 2.18 & AGN-1 & LEX \\
\hline $35 \ldots \ldots \ldots \ldots \ldots \ldots \ldots$ & $\ldots$ & $\ldots$ & $\ldots$ & $\ldots$ & $\ldots$ & $\ldots$ & $\ldots$ & $\ldots$ & $\ldots$ & $\ldots$ & $>4.84$ & $\ldots$ & $\ldots$ & $\ldots$ & $\ldots$ & $\ldots$ \\
\hline $36 \ldots \ldots \ldots \ldots \ldots \ldots$ & 0.076 & $\mathrm{~S}$ & 2.0 & $0.075 \pm 0.010$ & Uncl. & $0.16(0.01-0.31)$ & 2.33 & 18 & 22.18 & 40.20 & 0.06 & -2.39 & $<-0.79$ & $>2.65$ & Gal. & LEX \\
\hline 37. & 0.953 & V & 2.0 & $0.811 \pm 0.022$ & Galaxy & $1.01(0.75-1.27)$ & 2.33 & 18 & 23.67 & 41.72 & 0.90 & $<-1.63$ & $>-0.40$ & $\ldots$ & Uncl. & LEX \\
\hline 38 & 0.076 & $\mathrm{~S}$ & 2.0 & $0.086 \pm 0.012$ & Galaxy & $0.11(0.00-0.26)$ & 2.00 & 18 & 21.80 & 40.23 & -0.17 & -2.39 & -0.56 & 1.87 & Gal. & LEX \\
\hline 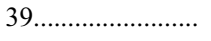 & $\ldots$ & $\ldots$ & $\ldots$ & $\ldots$ & $\ldots$ & $\ldots$ & $\ldots$ & $\ldots$ & $\ldots$ & $\ldots$ & 2.83 & $\ldots$ & $\ldots$ & $\ldots$ & $\ldots$ & $\ldots$ \\
\hline $40 \ldots \ldots \ldots \ldots \ldots \ldots$ & & $\ldots$ & $\ldots$ & $\ldots$ & $\ldots$ & $\ldots$ & $\ldots$ & $\ldots$ & $\ldots$ & $\ldots$ & 2.94 & $\ldots$ & $\ldots$ & $\cdots$ & $\ldots$ & $\ldots$ \\
\hline 41a & 2.223 & $\mathrm{~S}$ & 2.0 & $\ldots$ & $\ldots$ & $2.21(1.79-2.63)$ & 2.67 & 18 & 24.27 & 42.67 & 1.39 & -0.95 & $<0.02$ & $1.40^{\mathrm{b}}$ & AGNX & HEX \\
\hline $41 \mathrm{~b}$ & $\ldots$ & $\ldots$ & $\ldots$ & $0.841 \pm 0.055$ & Galaxy & $0.78(0.55-1.01)$ & 5.33 & 17 & 23.28 & $\ldots$ & 1.46 & $\ldots$ & $\ldots$ & $\ldots$ & $\ldots$ & $\ldots$ \\
\hline $42 \ldots \ldots \ldots \ldots \ldots \ldots$ & $\ldots$ & $\ldots$ & $\ldots$ & $\ldots$ & $\ldots$ & $\ldots$ & $\ldots$ & $\ldots$ & $\ldots$ & $\ldots$ & $>3.67$ & $\ldots$ & $\ldots$ & $\ldots$ & Uncl. & $\ldots$ \\
\hline
\end{tabular}


TABLE 2-Continued

\begin{tabular}{|c|c|c|c|c|c|c|c|c|c|c|c|c|c|c|c|c|}
\hline \multirow[b]{2}{*}{$\begin{array}{l}\text { No. } \\
\text { (1) }\end{array}$} & \multirow[b]{2}{*}{$\begin{array}{l}z_{\mathrm{sp}} \\
(2)\end{array}$} & \multirow[b]{2}{*}{$\begin{array}{l}\text { ORIGIN }^{a} \\
\text { (3) }\end{array}$} & \multirow[b]{2}{*}{$\begin{array}{l}Q_{z} \\
(4)\end{array}$} & \multicolumn{2}{|c|}{ COMBO-17 } & \multicolumn{3}{|c|}{ Mobasher ET AL. (2004) } & \multirow[b]{2}{*}{$\begin{array}{c}L_{1.4 \mathrm{GHz}} \\
\left(\mathrm{W} \mathrm{Hz}^{-1}\right) \\
(10)\end{array}$} & \multirow[b]{2}{*}{$\begin{array}{c}L_{\mathrm{X}} \\
\left(\mathrm{ergs} \mathrm{s}^{-1}\right) \\
(11)\end{array}$} & \multirow[b]{2}{*}{$\begin{array}{c}f_{1.4} / f_{\mathrm{opt}} \\
(12)\end{array}$} & \multirow[b]{2}{*}{$\begin{array}{c}f_{\mathrm{X}} / f_{\mathrm{opt}} \\
(13)\end{array}$} & \multirow[b]{2}{*}{$\begin{array}{l}\text { HR } \\
(14)\end{array}$} & \multirow[b]{2}{*}{$\begin{array}{c}\Gamma \\
(15)\end{array}$} & \multirow[b]{2}{*}{$\begin{array}{c}\text { Class (X-RAYS } \\
\text { or RADIO) } \\
(16)\end{array}$} & \multirow[b]{2}{*}{$\begin{array}{c}\text { Class } \\
\text { (OPtICAL) } \\
(17)\end{array}$} \\
\hline & & & & $\begin{array}{l}z_{\mathrm{ph}} \\
(5)\end{array}$ & $\begin{array}{c}\text { Type } \\
\text { (6) }\end{array}$ & $\begin{array}{l}z_{\mathrm{ph}} \\
(7)\end{array}$ & $\begin{array}{l}\text { Type } \\
\text { (8) }\end{array}$ & $\begin{array}{l}\text { No. of } \\
\text { Bands } \\
(9)\end{array}$ & & & & & & & & \\
\hline 43.......................... & $\ldots$ & $\ldots$ & $\ldots$ & & & & $\ldots$ & $\ldots$ & $\ldots$ & $\ldots$ & 3.54 & $\ldots$ & $\ldots$ & $\ldots$ & $\ldots$ & $\ldots$ \\
\hline 44......................... & 0.038 & $\mathrm{~S}$ & 2.0 & $0.058 \pm 0.006$ & Galaxy & $0.28(0.11-0.45)$ & 1.33 & 15 & 21.01 & 39.72 & -0.79 & $<-3.49$ & $>0.31$ & $<0.17$ & ULX & LEX \\
\hline 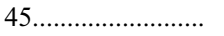 & $\ldots$ & $\ldots$ & $\ldots$ & $\ldots$ & $\ldots$ & $\ldots$ & $\ldots$ & $\ldots$ & $\ldots$ & $\ldots$ & 2.80 & $\ldots$ & $\ldots$ & $\ldots$ & $\ldots$ & $\ldots$ \\
\hline 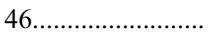 & 2.578 & $\mathrm{~S}$ & 2.0 & $\ldots$ & $\ldots$ & $2.51(2.05-2.97)$ & 2.00 & 14 & 24.48 & 42.62 & 2.16 & -0.32 & 0.30 & 0.19 & AGN-2 & HEX \\
\hline 47a & $\ldots$ & $\ldots$ & $\ldots$ & $0.528 \pm 0.031$ & Galaxy & $0.67(0.45-0.89)$ & 2.00 & 15 & 22.89 & $\ldots$ & 0.87 & $\ldots$ & $\ldots$ & $\ldots$ & $\ldots$ & $\ldots$ \\
\hline $47 \mathrm{~b}$ & $\ldots$ & $\ldots$ & $\ldots$ & $0.528 \pm 0.031$ & Galaxy & $0.67(0.45-0.89)$ & 2.00 & 15 & 22.89 & $\ldots$ & 0.74 & $\ldots$ & $\ldots$ & $\ldots$ & $\ldots$ & $\ldots$ \\
\hline 48a & $\ldots$ & $\ldots$ & $\ldots$ & $\ldots$ & $\ldots$ & $1.36(1.05-1.67)$ & 2.33 & 17 & 24.23 & $\ldots$ & 2.04 & $\ldots$ & $\ldots$ & $\ldots$ & $\ldots$ & $\ldots$ \\
\hline 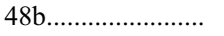 & $\ldots$ & $\ldots$ & $\ldots$ & $\ldots$ & $\ldots$ & $1.36(1.05-1.67)$ & 2.33 & 17 & 24.23 & $\ldots$ & 2.02 & $\ldots$ & $\ldots$ & $\ldots$ & $\ldots$ & $\ldots$ \\
\hline 49......................... & 1.603 & $\mathrm{~S}$ & 2.0 & $1.015 \pm 0.127$ & Galaxy & $1.76(1.40-2.12)$ & 1.67 & 14 & 24.10 & 44.30 & 1.82 & 1.31 & -0.51 & 1.75 & QSO-1 & HEX \\
\hline $50 \ldots \ldots \ldots \ldots \ldots \ldots \ldots$ & $\ldots$ & $\ldots$ & $\ldots$ & $0.567 \pm 0.032$ & Galaxy & $0.58(0.37-0.79)$ & 2.67 & 18 & 22.93 & $\ldots$ & 0.91 & $\ldots$ & $\ldots$ & $\ldots$ & $\ldots$ & $\ldots$ \\
\hline 51a................................ & $\ldots$ & $\ldots$ & $\ldots$ & $0.652 \pm 0.012$ & Galaxy & $0.61(0.40-0.82)$ & 1.00 & 15 & 23.32 & $\ldots$ & 0.76 & $\ldots$ & $\ldots$ & $\ldots$ & $\ldots$ & $\ldots$ \\
\hline $51 \mathrm{~b} \ldots \ldots \ldots \ldots \ldots \ldots \ldots \ldots$ & $\ldots$ & $\ldots$ & $\ldots$ & $0.650 \pm 0.014$ & Galaxy & $0.61(0.40-0.82)$ & 1.00 & 15 & 23.32 & $\ldots$ & 0.70 & $\ldots$ & $\ldots$ & $\ldots$ & $\ldots$ & $\ldots$ \\
\hline 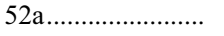 & 3.064 & $\mathrm{~S}$ & 2.0 & $\ldots$ & $\ldots$ & $1.17(0.89-1.45)$ & 2.33 & 13 & 24.61 & 43.83 & 2.08 & 0.81 & 0.18 & 0.42 & AGN-2 & HEX \\
\hline $52 \mathrm{~b} \ldots \ldots \ldots \ldots \ldots \ldots \ldots \ldots \ldots$ & $\ldots$ & $\ldots$ & $\ldots$ & $1.400 \pm 0.076$ & Galaxy & $0.85(0.61-1.09)$ & 1.33 & 14 & 23.82 & $\ldots$ & 1.28 & $\ldots$ & $\ldots$ & $\ldots$ & $\ldots$ & $\ldots$ \\
\hline $53 \ldots \ldots \ldots \ldots \ldots \ldots$ & 0.625 & $\mathrm{~S}$ & 0.5 & $\ldots$ & $\ldots$ & $1.81(1.44-2.20)$ & 2.00 & 11 & 23.18 & 42.76 & 2.52 & 0.93 & 0.40 & -0.02 & AGN-2 & ABS \\
\hline 54 & & & & & & $1.63(1.28-1.99)$ & 2.67 & 14 & 24.00 & $\ldots$ & 1.88 & $\ldots$ & $\ldots$ & $\ldots$ & $\ldots$ & $\ldots$ \\
\hline $55 \mathrm{a}$ & 0.279 & $\mathrm{~S}$ & 1.0 & $0.264 \pm 0.022$ & Galaxy & $0.46(0.27-0.65)$ & 1.33 & 15 & 23.07 & $\ldots$ & 0.54 & $\ldots$ & $\ldots$ & $\ldots$ & $\ldots$ & ABS \\
\hline $55 \mathrm{~b}$ & 0.279 & $\mathrm{~S}$ & 2.0 & $0.262 \pm 0.049$ & Galaxy & $0.28(0.11-0.45)$ & 1.33 & 15 & 23.07 & 41.33 & 0.82 & -1.51 & $<-0.72$ & $>2.35$ & Gal. & HEX/Sey2 \\
\hline 56 & 0.214 & $\mathrm{~S}$ & 2.0 & $0.187 \pm 0.031$ & Galaxy & $0.37(0.19-0.55)$ & 2.33 & 16 & 22.14 & $\ldots$ & -0.15 & $\ldots$ & $\ldots$ & $\ldots$ & $\ldots$ & LEX \\
\hline 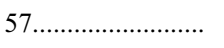 & 0.458 & $\mathrm{~S}$ & 2.0 & $0.467 \pm 0.008$ & Galaxy & $0.45(0.26-0.64)$ & 2.33 & 15 & 22.94 & 41.38 & 0.12 & -2.14 & $<-0.09$ & $1.40^{\mathrm{b}}$ & Uncl. & LEX \\
\hline $58 \ldots \ldots \ldots \ldots \ldots \ldots \ldots \ldots \ldots$ & $\ldots$ & $\ldots$ & $\ldots$ & $\ldots$ & $\ldots$ & $\ldots$ & $\ldots$ & $\ldots$ & $\ldots$ & $\ldots$ & $>4.06$ & $\ldots$ & $\ldots$ & $\ldots$ & $\ldots$ & $\ldots$ \\
\hline 59 & $\ldots$ & $\ldots$ & $\ldots$ & $0.833 \pm 0.054$ & Galaxy & $1.40(1.08-1.72)$ & 1.67 & 11 & 23.37 & $\ldots$ & 1.80 & $\ldots$ & $\ldots$ & $\ldots$ & $\ldots$ & $\ldots$ \\
\hline 60a............................... & $\ldots$ & $\ldots$ & $\ldots$ & $1.065 \pm 0.106$ & Galaxy & $1.25(0.89-1.55)$ & 3.67 & 15 & 23.62 & $\ldots$ & 1.76 & $\ldots$ & $\ldots$ & $\ldots$ & $\ldots$ & $\ldots$ \\
\hline $60 \mathrm{~b}$ & $\ldots$ & $\ldots$ & $\ldots$ & $\ldots$ & $\ldots$ & $0.17(0.02-3.10)$ & 3.67 & 13 & 21.79 & $\ldots$ & 2.25 & $\ldots$ & $\ldots$ & $\ldots$ & $\ldots$ & $\ldots$ \\
\hline 61....................... & $\ldots$ & $\ldots$ & $\ldots$ & $\ldots$ & $\ldots$ & & $\ldots$ & $\ldots$ & $\ldots$ & $\ldots$ & 3.08 & $\ldots$ & $\ldots$ & $\ldots$ & $\ldots$ & $\ldots$ \\
\hline 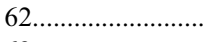 & 1.117 & $\mathrm{~L}$ & 0.5 & $0.987 \pm 0.105$ & Galaxy & $1.06(0.79-1.33)$ & 1.00 & 9 & 23.76 & $\ldots$ & 1.80 & -0.56 & $\ldots$ & $\ldots$ & Uncl. & $\ldots$ \\
\hline 63.................... & $\ldots$ & $\cdots$ & $\ldots$ & $0.816 \pm 0.059$ & Galaxy & $0.87(0.62-1.12)$ & 2.00 & 13 & 23.34 & $\ldots$ & 1.00 & $\ldots$ & $\ldots$ & $\ldots$ & $\ldots$ & $\ldots$ \\
\hline 64............................ & $\ldots$ & $\ldots$ & $\ldots$ & $\ldots$ & $\ldots$ & $0.45(0.25-1.35)$ & 2.00 & 10 & 22.74 & 42.05 & 2.40 & 1.01 & -0.55 & 1.81 & AGN-1 & $\ldots$ \\
\hline
\end{tabular}

Noте.-Table 2 is also available in machine-readable form in the electronic version of the Astronomical Journal.

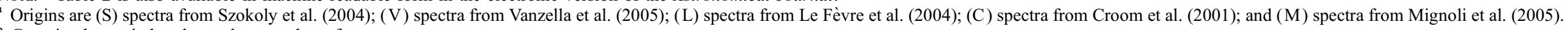

b Generic photon index due to low number of counts.

${ }^{c} \mathrm{HR}$ as given in Giacconi et al. (2002), calculated using the $0.5-2$ and $2-10 \mathrm{keV}$ bands. 
is the spectroscopic quality flag. Given the different sources for spectroscopic information, we have converted quality flags from Le Fèvre et al. (2004), Vanzella et al. (2005), and Mignoli et al. (2005) to a scale similar to the one in Szokoly et al. (2004); namely, $Q_{z}=2.0$ denotes a reliable redshift determination, $Q_{z}=1.0$ indicates that some spectroscopic feature exists but cannot be securely identified, and $Q_{z}=0.5$ flags sources for which there is only a hint of a spectral feature. Column (5) is the photometric redshift from the COMBO-17 work (Wolf et al. 2004). Column (6) is the classification from the COMBO-17 work (Wolf et al. 2004). Column (7) is the photometric redshift and associated 95\% confidence interval from Mobasher et al. (2004). Column (8) is the bestfit spectral galaxy type $(E=1, \mathrm{Sbc}=2, \mathrm{Scd}=3, \mathrm{IM}=4$, and starburst $=5$, 6) from Mobasher et al. (2004). Column (9) is the number of bands in which the object is detected from Mobasher et al. (2004). Column (10) is the logarithm of the $1.4 \mathrm{GHz}$ luminosity in $\mathrm{W} \mathrm{Hz}^{-1}$. Column (11) is the logarithm of the X-ray (0.5$8 \mathrm{keV}$ ) luminosity in ergs $\mathrm{s}^{-1}$. Column (12) is the radio-to-optical flux ratio. Column (13) is the X-ray-to-optical flux ratio. Column (14) is the HR, derived from the $0.5-2$ and $2-8 \mathrm{keV}$ band counts. For sources detected only in the Giacconi et al. (2002) catalogs, we report the quoted HR, derived from the $0.5-2$ and 2$10 \mathrm{keV}$ band counts, clearly marking such cases. Column (15) is the effective photon index $\Gamma$, as given by Alexander et al. (2003). Column (16) is the classification obtained from the X-ray characteristics ( $L_{X}$ and/or HR) or, if possible for sources with insufficient $\mathrm{X}$-ray information, from the radio power. Column (17) is the classification obtained from the optical spectrum, as in Szokoly et al. (2004), or after estimating any possible line-emission diagnostic ratios (separating Seyfert 2 galaxies [Sey2] and star-forming galaxies $[\mathrm{SF}])$.

\section{COMMENTS ON INDIVIDUAL SOURCES}

1. ATCDFS J033159.86-274541.3: Unidentified at optical $\left(z_{850}>27.7 \mathrm{mag}\right.$ and undetected in the stacked $B_{435}+$ $V_{606}+i_{775}+z_{850}$ image) and X-ray wavelengths.

2. ATCDFS J033204.81-274125.8: The optical counterpart matches the position of the X-ray identification. It presents a complex optical morphology, with several peaks of brightness within a 0 . $5 \times 0$ ".5 region. The spectroscopic redshift $\left(z_{\mathrm{sp}}=0.720\right)$, based on a single emission line considered to be $\mathrm{Mg}$ II, is supported by the photometric redshift estimate. The high X-ray luminosity and hard X-ray spectrum indicate a type 2 AGN.

3. ATCDFS J033205.07-274535.5: A faint, extended optical object is observed 2".6 away from the radio position, at the border of the $3 \sigma$ radio position error region. The estimated photometric redshift is highly uncertain owing to the small number of optical bands in which the object is detected. No X-ray emission is observed.

4. ATCDFS J033208.51-274648.8: A bright $\left(z_{850}=\right.$ $18.6 \mathrm{mag}$ ) disk galaxy at $z_{\mathrm{sp}}=0.310$, also detected in the X-ray. Optical spectroscopic line ratios indicate the presence of an AGN, confirmed by the X-ray emission properties ( $L_{X}$ and HR), despite the low $\mathrm{X}$-ray-to-optical flux ratio (source undetected in the soft $0.5-2 \mathrm{keV}$ X-ray band).

5. ATCDFS J033208.60-274043.0: An optically bright $\left(z_{850}=20.0 \mathrm{mag}\right)$ disk galaxy at $z_{\mathrm{ph}}=0.35$. An optically unidentified X-ray detection exists just outside the radio $3 \sigma$ error region, but it seems unrelated to the radio emission.

6. ATCDFS J033208.67-274734.3: An optical pointlike source at $z_{\mathrm{sp}}=0.543$, showing indications of underlying lowlevel extended optical emission. The implied high X-ray luminosity and hard X-ray spectrum classify this as a type 1 QSO. Broad emission lines are detected in the optical spectrum.
7. ATCDFS J033209.72-274249.0: A spheroidal galaxy also detected in X-rays, at $z_{\mathrm{sp}}=0.733$. The X-ray emission is characteristic of a type 1 AGN. Two nearby (within $20^{\prime \prime}$ ) galaxies show similar redshifts, at $z_{\mathrm{sp}}=0.728$ and 0.729 . This is one of the members of the structure found at $z \sim 0.73$ in the CDF-S (Gilli et al. 2003; Szokoly et al. 2004; Adami et al. 2005).

8. ATCDFS J033210.80-274629.2: A compact optical source also detected in X-rays. The redshift $\left(z_{\mathrm{sp}}=1.610\right)$ implies high $\mathrm{X}$-ray and radio luminosities characteristic of an $\mathrm{AGN}$, as does the X-ray-to-optical flux ratio.

9. ATCDFS J033210.91-274415.1: A broad emission line type 1 QSO at $z_{\mathrm{sp}}=1.615$ as revealed by the optical spectroscopy and X-ray properties. Extended emission from the host galaxy is visible in the ACS images.

10. ATCDFS J033211.00-274053.6: A compact optical source, also displaying X-ray emission, close (3".5 away) to a bright spiral galaxy. The absence of a spectroscopic or a photometric redshift prevents classification of this object, although the $\mathrm{X}$-ray-to-optical flux ratio is indicative of an AGN. The observed properties of this source are similar to those of the type 1 QSO identified for source 9, perhaps at a higher redshift.

11. ATCDFS J033211.51-274711.5: A galaxy with a highly irregular optical morphology at $z_{\mathrm{sp}}=0.576$. No X-ray emission is detected.

12. ATCDFS J033213.08-274351.0: Despite being a relatively bright radio source in this sample $\left(S_{1.4 \mathrm{GHz}}=1.4 \mathrm{mJy}\right)$, there is no likely optical $\left(z_{850}>28.2 \mathrm{mag}\right.$ and undetected in the stacked $B_{435}+V_{606}+i_{775}+z_{850}$ image) or X-ray counterpart. The radio-to-optical flux ratio $(>4.86)$ is one of the largest in this sample.

13. ATCDFS J033213.28-274240.3: A complex system apparently involving a bright $\left(z_{850}=19.7 \mathrm{mag}\right)$ galaxy (source 13a), with faint spiral structure surrounded by several knots of optical emission, and a possible edge-on galaxy (source 13b). This possible merger system is identified as a single object in the public GOODS ACS catalog; SExtractor was used to deblend the two major components. The brightest source (13a) has an X-ray detection at a redshift of $z_{\mathrm{sp}}=0.605$, with X-ray properties characteristic of a type 2 AGN.

14. ATCDFS J033214.17-274910.2: A faint $\left(z_{850}=\right.$ $24.1 \mathrm{mag}$ ), extended object with a redshift of $z_{\mathrm{ph}}=1.66$ and no $\mathrm{X}$-ray detection.

15. ATCDFS J033217.11-274303.9: A spheroidal galaxy at $z_{\text {sp }}=0.569$, with optical spectroscopy indicating a broad emission line AGN and the X-ray emission classifying it as a type 1 AGN.

16. ATCDFS J033217.22-275222.4: The likely optical counterpart is a disk galaxy at $z_{\mathrm{sp}}=1.097$ with X-ray emission indicative of a type 2 AGN. The radio emission extends to the southeast, toward a brighter source with a similar spectroscopic redshift of $z_{\mathrm{sp}}=1.098$. The spectroscopic observations available in a $1^{\prime} \times 1^{\prime}$ region centered in this source reveal eight redshifts between 1.05 and 1.10. This indicates the existence of a high-redshift complex likely also including radio source 20 . In fact, a massive group has recently been detected in the CDF-S at this very redshift (Adami et al. 2005).

17. ATCDFS J033218.01-274718.4: A galaxy at $z_{\mathrm{sp}}=$ 0.734, also detected in the X-ray. Both optical spectroscopy and $\mathrm{X}$-ray properties indicate a star-forming galaxy, although at high $\mathrm{X}$-ray and radio luminosities. The ACS image actually resolves this source into a brighter spheroidal component and a much fainter companion 0".5 away, likely revealing a merging event. Five nearby (within $30^{\prime \prime}$ ) sources have similar spectroscopic redshifts $\left(z_{\mathrm{sp}}=0.73-0.74\right)$. These are members of the structure 
known to exist in the CDF-S at this redshift (Gilli et al. 2003; Szokoly et al. 2004; Adami et al. 2005).

18. ATCDFS J033218.03-275056.2: A faint optical source with a complex morphology lies inside the radio position error region. The photometric redshift $\left(z_{\mathrm{ph}}=1.04\right)$, essentially from ground-based photometry, is likely to be biased by a slightly brighter source 0 ". 4 to the south, whose relation to the assumed optical identification is unclear. A nearby bright disk galaxy and the X-ray detection east of the radio coordinates are too distant to be considered related to the radio emission.

19. ATCDFS J033218.83-275410.3: No optical identification exists within the $3 \sigma$ radio position error region. Instead, this radio source is thought to form, with source 21 , the double radio lobe structure related to a cD-type galaxy 10"2 away (bright galaxy indicated by the horizontal lines), at a photometric redshift of $z_{\mathrm{ph}}=0.96$. A weak X-ray detection from the Giacconi et al. (2002) catalog is associated with the optical galaxy, indicating the presence of a type 2 AGN. From the available redshift surveys in this field, there are four nearby galaxies at similar redshifts $\left(z_{\mathrm{sp}}=0.97-1.04\right)$, which indicates the presence of a group. The association with another weak X-ray detection, $3^{\prime \prime}$ south from the radio position (see Fig. 2) and again detected only in the Giacconi et al. (2002) catalog (identified as source 527 in that catalog), is unclear.

20. ATCDFS J033219.41-275216.5: An irregular galaxy showing indications for a tidal tail toward the southeast, which suggests a merger event. The photometric redshift $z_{\mathrm{ph}}=1.05$ is comparable to the spectroscopic redshift of 1.096 for the slightly fainter galaxy $3^{\prime \prime}$ to the southwest, a possible merger companion. The redshifts suggest membership of the structure discussed for source 16.

21. ATCDFS J033219.57-275403.2: This source is thought to be the second radio lobe associated with a cD-type galaxy 5".5 away (the bright galaxy indicated by the horizontal lines). See the discussion for source 19, the other proposed radio lobe.

22. ATCDFS J033219.82-274121.2: A galaxy at $z_{\mathrm{sp}}=$ 0.229 , also detected in the X-ray. The X-ray emission does not provide a definite classification of this source, although the optical line ratio points to a Seyfert 2-type galaxy. The X-ray-to-optical flux ratio, however, is more indicative of star formation.

23. ATCDFS J033221.24-274436.1: The optical identification seems to be part of a merging pair, with the second galaxy just outside the radio position error region. The optical spectrum, revealing a redshift of $z_{\mathrm{sp}}=0.524$, likely includes light from both sources, 0 ". 9 apart. This is also a weak X-ray detection in the $0.5-$ $1 \mathrm{keV}$ band (source S12 of Alexander et al. [2003]). The low $\mathrm{X}$-ray-to-optical flux ratio is indicative of star formation.

24. ATCDFS J033221.92-274243.8: A weak extended optical source. The photometric redshift of $z_{\mathrm{ph}}=1.54$ may be biased by a brighter source 1 " to the north, whose relation to the assumed optical identification is unclear.

25. ATCDFS J033222.36-274807.3: Unidentified at opti$\operatorname{cal}\left(z_{850}>28.4\right.$ mag and undetected in the stacked $B_{435}+V_{606}+$ $i_{775}+z_{850}$ image) and X-ray wavelengths. The nearby X-ray detections seem unrelated to the radio emission.

26. ATCDFS J033222.64-274424.5: A spiral galaxy at $z_{\mathrm{sp}}=$ 0.737 , displaying weak X-ray emission detected only in the $0.5-$ $8 \mathrm{keV}$ band (source 148 of Alexander et al. [2003]). The optical spectroscopy, although of low $\mathrm{S} / \mathrm{N}$, is indicative of star formation, as is the low X-ray-to-optical flux ratio. This is another likely member of the structure detected at this redshift in the CDF-S (Gilli et al. 2003; Szokoly et al. 2004; Adami et al. 2005).

27. ATCDFS J033224.59-275441.6: A disk galaxy at $z_{\mathrm{sp}}=0.123$, with no X-ray emission. The available optical spec- troscopy does not provide enough lines to apply line ratio diagnostics.

28. ATCDFS J033225.15-275452.2: An ellipsoidal galaxy with a spectroscopic redshift of $z_{\mathrm{sp}}=1.090$, also detected in the $\mathrm{X}$-ray. The X-ray emission classifies it as a type 2 AGN, while the optical spectroscopy reveals an absorption-line spectrum. This is a likely member of the structure recently found at $z \sim$ 1.10 in the CDF-S (Adami et al. 2005).

29. ATCDFS J033226.98-274106.5: This is the brightest radio source in the ACS area, with $S_{1.4 \mathrm{GHz}}=20 \mathrm{mJy}$. The optical identification is a pointlike source that shows the brightest X-ray flux in this sample. At $z_{\mathrm{sp}}=0.734$, this is a type $1 \mathrm{QSO}$, with optical spectroscopy revealing a broad-line AGN. At least three other galaxies lie at the same redshift in the surrounding $1^{\prime} \times 1^{\prime}$ region, again tracing the structure detected at this redshift in the CDF-S (Gilli et al. 2003; Szokoly et al. 2004; Adami et al. 2005).

30. ATCDFS J033227.64-275040.5: A galaxy at $z_{\mathrm{sp}}=$ 1.097, also detected in X-rays. The optical image reveals a very irregular, knotty morphology, possibly indicative of a merging event and related star formation. The X-ray emission does not clarify the processes behind its luminosity, although the X-rayto-optical flux ratio is typical of star-forming galaxies. The optical spectroscopy presents some lines that are more common in AGNs (e.g., [Ne v] 23425). However, this line may also be generated in shocks in interacting galaxies. This radio source is another likely member of the massive group recently detected in the CDF-S at $z \sim 1.10$ (Adami et al. 2005).

31. ATCDFS J033227.98-274641.5: A bright $\left(z_{850}=\right.$ $19.4 \mathrm{mag}$ ) disk galaxy at $z_{\mathrm{sp}}=0.247$. This source was observed spectroscopically by Croom et al. (2001), revealing a spectrum dominated by absorption features, although it presents emission in $\mathrm{H} \alpha$ and [N II]. A fainter optical source, which could be a companion galaxy, is seen 1".5 to the north.

32. ATCDFS J033228.31-273842.9: A galaxy at $z_{\mathrm{ph}}=0.39$ presenting irregular structure with a nearby (1."7 away), possibly related companion.

33. ATCDFS J033228.71-274402.3: Unidentified at optical $\left(z_{850}>28.1 \mathrm{mag}\right.$ and undetected in the stacked $B_{435}+V_{606}+$ $i_{775}+z_{850}$ image) and X-ray wavelengths.

34. ATCDFS J033228.78-274620.8: An ellipsoidal galaxy at a redshift of $z_{\mathrm{sp}}=0.738$, also detected in X-rays. Several nearby fainter optical sources exist, and low-level optical emission bridges to at least one neighbor, which suggests interaction. The galaxy is classified as a type $1 \mathrm{AGN}$ from its X-ray properties. Again, this is a likely member of the $z \sim 0.73$ structure that exists in the CDF-S (Gilli et al. 2003; Szokoly et al. 2004; Adami et al. 2005).

35. ATCDFS J033229.59-274332.5: Unidentified at optical $\left(z_{850}>28.1 \mathrm{mag}\right.$ and undetected in the stacked $B_{435}+V_{606}+$ $i_{775}+z_{850}$ image) and X-ray wavelengths.

36. ATCDFS J033229.83-274423.7: A flocculent galaxy, with X-ray emission coincident with its nuclear region. At $z_{\mathrm{sp}}=$ 0.076 the galaxy is part of a larger structure; low-level optical emission connects this galaxy to the optical identification of source 38 , indicating an interacting pair (as previously noted by Giacconi et al. 2001), and a smaller galaxy at the same redshift is located roughly $20^{\prime \prime}$ west. The X-ray characteristics indicate a star-forming galaxy.

37. ATCDFS J033229.89-274520.0: A spiral/flocculent galaxy at $z_{\mathrm{sp}}=0.953$. X-ray emission detected toward the west side of the galaxy could be related to an area of brighter optical emission in the western spiral arm. The X-ray and radio characteristics do not reveal AGN activity. Two other galaxies in the immediate vicinity (within $9^{\prime \prime}$ ) lie at similar redshifts, indicating the presence of a larger structure. 
38. ATCDFS J033229.96-274404.8: Another flocculent galaxy at $z_{\mathrm{sp}}=0.076$, connected by low-level optical emission to source 36. X-ray emission is detected north of the nuclear region, with characteristics indicative of star formation.

39. ATCDFS J033229.98-275258.7: An extended faint optical source $\left(z_{850}=26.19\right)$ is identified as the possible optical counterpart. However, this is a complex region, in both the optical and the radio; a couple of faint optical sources appear near the radio position in the combined $B_{435}+V_{606}+i_{775}+z_{850}$ image, and the radio emission partly overlaps source 40 . In fact, an $\mathrm{X}$-ray source associated with an optically complex multiple system is observed halfway between sources 39 and 40. A possible interpretation would be that these two radio sources are somehow associated with the X-ray source (source S22 of Alexander et al. [2003]), although the X-ray characteristics and optical spectroscopy do not indicate any AGN activity.

40. ATCDFS J033230.20-275312.7: The most likely optical counterpart is a very faint $\left(z_{850}=26.8 \mathrm{mag}\right)$ galaxy revealed in the combined $B_{435}+V_{606}+i_{775}+z_{850}$ image as a pair of sources. It should be noted that this is a complex region in both the optical and the radio, with some overlapping between the radio emission of sources 39 and 40, which could even be related; see the discussion for source 39 .

41. ATCDFS J033231.44-274621.5: The radio source has two candidate optical identifications with similar values of $\mathcal{R}$. The most likely (source 41a) is an X-ray source with a spectroscopic redshift $z_{\mathrm{sp}}=2.223$, which agrees with the photometric redshift estimate. This source has a high X-ray luminosity, indicating an AGN (see also Szokoly et al. 2004), although Daddi et al. (2004) argue that it may be a vigorous star-forming galaxy. Recently, van Dokkum et al. (2005) performed Gemini near-infrared spectroscopy of this source and found a surprising lack of AGN spectral features. Instead, the observed Seyfert-like line ratios could be explained by shock ionization due to a strong galactic wind in a starburst galaxy. A second possible identification (source 41b) has no detectable X-ray emission and a photometric redshift estimate of $z_{\mathrm{ph}}=0.84$. The optical image shows an extended galaxy, hinting at the existence of tidal tail disruption.

42. ATCDFS J033233.44-275228.0: Unidentified at optical wavelengths $\left(z_{850}>28.1 \mathrm{mag}\right.$ and undetected in the stacked $B_{435}+V_{606}+i_{775}+z_{850}$ image). A weak X-ray detection exists in the Giacconi et al. (2002) catalog.

43. ATCDFS J033234.97-275456.1: A faint object is just perceptible in the $z_{850}$ image, although it is not present in the public GOODS ACS catalogs. In the stacked $B_{435}+V_{606}+i_{775}+$ $z_{850}$ image, two faint objects are visible. Inspection of the individual optical bands reveals one of these sources appearing only in the bluer bands ( $B_{435}$ and $V_{606}$ ), while the other appears mainly in the $V_{606}$ and $i_{775}$ bands, being just hinted at in the redder band at $z_{850} \sim 27.5$ (value from aperture photometry).

44. ATCDFS J033235.07-275532.8: A disk galaxy at $z_{\text {sp }}=$ 0.038 with X-ray emission originating from the disk. As discussed by Szokoly et al. (2004), the X-ray luminosity, HR, and location of the X-ray emission indicate an ultraluminous X-ray source (ULX) hosted by a normal galaxy. The low radio luminosity is consistent with this interpretation.

45. ATCDFS J033235.46-275452.8: A faint optical source $\left(z_{850}=26.8 \mathrm{mag}\right)$ is identified as the possible counterpart, although the stacked $B_{435}+V_{606}+i_{775}+z_{850}$ image hints at the presence of fainter sources in the region. The optical counterpart candidate displays a faint elongated morphology. No X-ray emission is present in this region.

46. ATCDFS J033235.71-274916.0: A compact optical source with a close neighbor only visible in the ACS images (0". 3 away) is the probable identification, at $z_{\mathrm{sp}}=2.578$. X-ray emission is also present. The radio and X-ray properties classify this as a type 2 AGN.

47. ATCDFS J033237.22-275129.7: A pair of interacting galaxies at a photometric redshift, which includes light from both sources, of $z_{\mathrm{ph}}=0.53$. The western galaxy is a face-on spiral (source 47a), while the eastern source is a disk galaxy (source 47b). No X-ray emission is detected.

48. ATCDFS J033237.75-275000.7: A complex optical system at $z_{\mathrm{ph}}=1.36$, with the photometric redshift estimate including light from the entire region. Two optical sources separated by around 1 " are identified as likely counterparts. Careful inspection of the stacked $B_{435}+V_{606}+i_{775}+z_{850}$ image reveals four patches of optical emission surrounded by elongated structures of low surface brightness, perhaps indicating a merger event. No $\mathrm{X}$-ray emission is detected.

49. ATCDFS J033237.75-275211.9: A compact optical source also detected in the X-ray with $z_{\mathrm{sp}}=1.603$. The $\mathrm{X}$-ray properties (luminosity and HR) indicate a type 1 QSO.

50. ATCDFS J033238.75-274632.5: The possible optical identification for this weak radio source is a bright $\left(z_{850}=\right.$ $21.533 \mathrm{mag}$ ) galaxy at $z_{\mathrm{ph}}=0.57$. However, we note that this optical identification is of low reliability $(\mathcal{R}=0.23)$, and the slightly extended east-west radio contours raise the possibility of complex radio emission originating in more than one of the galaxies seen in this region.

51. ATCDFS J033239.46-275300.9: Two ellipsoidal galaxies separated by 1.2 in a likely merging event (as indicated by the low surface brightness extended emission). The photometric redshift is $z_{\mathrm{ph}}=0.65$, identical for both sources but resulting from separate analyses in the Wolf et al. (2004) work.

52. ATCDFS J033239.64-274851.8: Two unrelated optical sources are possible counterparts. The most likely counterpart (source 52a) is an X-ray source with $z_{\mathrm{sp}}=3.064$, classified as a type 2 AGN from its X-ray properties. The other possible counterpart (source 52b) has no X-ray emission and a photometric redshift of $z_{\mathrm{ph}}=1.40$.

53. ATCDFS J033240.82-275545.8: A faint optical source $\left(z_{850}=25.18 \mathrm{mag}\right)$ with X-ray emission. A spectrum with a "hint of some spectral feature" was obtained for this source by Szokoly et al. (2004), suggesting a redshift of $z_{\mathrm{sp}}=0.625$. The photometric estimate results in a higher value of $z_{\mathrm{ph}}=1.81$. Considering even the smallest (spectroscopic) value, the X-ray characteristics indicate a type $2 \mathrm{AGN}$ consistent with the X-rayto-optical flux ratio.

54. ATCDFS J033243.12-275514.2: A pair of galaxies separated by $0 . " 6$ with disturbed morphology associated with traces of low surface brightness tails, indicating a merging system. The photometric redshift, based on the light from both sources, is $z_{\mathrm{ph}}=1.63$. No X-ray emission is detected.

55. ATCDFS J033244.16-275142.4: A spectroscopically confirmed pair of galaxies at $z_{\mathrm{sp}}=0.279$. The western companion (source 55a) is orientated edge-on and has clear signs of dust obscuration, displaying an optical spectrum dominated by absorption features. The eastern galaxy (source 55b) displays an emission-line optical spectrum with inconclusive line ratios. This latter galaxy is an X-ray source, with X-ray characteristics indicative of star formation. However, optical spectroscopy reveals line ratios suggesting a Seyfert 2-type galaxy.

56. ATCDFS J033244.93-274726.2: A spiral galaxy at $z_{\mathrm{sp}}=$ 0.214 with no X-ray emission and a narrow emission-line optical spectrum.

57. ATCDFS J033245.03-275438.8: A face-on spiral galaxy with $z_{\mathrm{sp}}=0.458$, showing knots of star formation. Faint X-ray 
emission is detected, possibly off-center toward the north arm of the galaxy. The X-ray characteristics are consistent with star formation.

58. ATCDFS J033246.03-275318.2: No reliable optical counterpart is detected for this radio source, even in the stacked $B_{435}+V_{606}+i_{775}+z_{850}$ image. No X-ray emission is observed either. One should note that this is a complex region in the radio, since the radio emission overlaps with that of source 59.

59. ATCDFS J033246.33-275328.6: The likely optical counterpart exhibits a complex morphology and an estimated photometric redshift of $z_{\mathrm{ph}}=0.833$. No X-ray emission is detected.

60. ATCDFS J033246.78-275120.1: Two galaxies without $\mathrm{X}$-ray emission are possible optical counterparts, with similar values for $\mathcal{R}$. Photometry indicates $z_{\mathrm{ph}}=1.06$ for the southern object (source 60a), while the northern galaxy (source 60b) has no reliable redshift determination.

61. ATCDFS J033248.02-275414.7: A faint optical source $\left(z_{850}=26.57 \mathrm{mag}\right)$ lies close to the radio position and is the possible optical counterpart. The faint magnitude prevents any photometric redshift estimate.

62. ATCDFS J033248.62-274934.9: An extended object at a spectroscopic redshift of $z_{\mathrm{sp}}=1.117$, which despite being based on a low $\mathrm{S} / \mathrm{N}$ spectrum is supported by photometric redshift estimates. A second optical source lies less than $1^{\prime \prime}$ to the southeast, just outside the $3 \sigma$ radio position error region. Traces of low surface brightness emission connect both sources, which suggests an interacting system. This is also a weak X-ray detection in the Giacconi et al. (2002) catalog.

63. ATCDFS J033300.91-275049.4: A bright $\left(z_{850}=\right.$ $21.67 \mathrm{mag}$ ) optical source is identified as the possible optical counterpart, although at a relatively low value of $\mathcal{R}$. No X-ray emission is detected from this region.

64. ATCDFS J033302.99-275147.3: An X-ray counterpart exists with a weak optical source $\left(z_{850}=25.13 \mathrm{mag}\right)$ lying just outside the position error box of the X-ray detection. The photometric redshift of the optical source is $z_{\mathrm{ph}}=0.45$, as estimated by Mobasher et al. (2004). However, for this same optical source, Zheng et al. (2004) find $z_{\mathrm{ph}}=3.69$ using other photometric redshift codes. If the optical and X-ray emission are from the same source, the X-ray characteristics indicate, at the lowest redshift mentioned above, a type 1 AGN. If the higher redshift is the correct one, then this is classified as a type 1 QSO.

\section{DISCUSSION}

There are 64 sources in the radio catalog. Likely ACS counterparts have been identified for 50 of these $(78 \%$, where we include unique optical counterparts with $\mathcal{R} \geq 0.5$, likely merger systems, and sources 19 and 21 as the radio lobes of the same radio galaxy). Optical identifications with lower reliability $(\mathcal{R}<$ 0.5 ) exist for seven radio sources, including three cases in which seemingly independent optical identifications are equally reliable at the $\mathcal{R} \sim 0.5$ level (sources 41, 52, and 60). For seven radio sources $(11 \%)$, no optical identification was possible.

The redshifts for the optically identified radio sources range from below 0.1 to above 3 , although most are found up to $z \sim 1.1$. This is in agreement with previous work on the HDF-N and the Small Selected Area 13 fields, which reach similar depths for the spectroscopic observations (Chapman et al. 2003). Several radio sources are identified at $z \sim 0.73$ and $z \sim 1.10$. These are likely members of the structures already found in the CDF-S region (Gilli et al. 2003; Szokoly et al. 2004; Adami et al. 2005).

A total of 34 radio sources $(53 \%)$ have an X-ray counterpart; in all but three cases, the $\mathrm{X}$-ray identifications are unambigu- ously associated with reliable optical counterparts of the radio sources. For sources 41 and 52, for which two optical sources appear as possible counterparts, the X-ray detection suggests which optical counterpart is the real one. Source 42 has no optical counterpart despite the X-ray detection.

The seven radio sources without ACS counterparts (hereafter "optically faint radio sources") are of particular interest. In a pioneering study of optically faint submillijansky radio sources, Richards et al. (1999) reported that approximately $60 \%$ of the radio sources detected in very deep 1.4 GHz surveys of the HDF-N and the Small Selected Area 13 fields have relatively bright $(I \sim$ 22 mag) disk galaxy counterparts, $20 \%$ have low-luminosity AGN counterparts, and the remaining $20 \%$ have no counterpart brighter than $I=25$. Deep $K$-band imaging of this population revealed that several are very red objects, with $I-K>4$ (Richards et al. 1999), suggesting that these objects form an inhomogeneous population, including high-redshift dusty starbursts, extreme-redshift, radio-loud AGNs, moderate-redshift AGNs with optical hosts having a relatively low optical power, or one-sided radio jets. With the deep ACS imaging reported here, we find 16 sources $(25 \%)$ with $z_{850} \geq 25$ mag, with the fraction of optically unidentified $\left(z_{850} \gtrsim\right.$ $28 \mathrm{mag}$ ) radio sources being $11 \%$ (seven sources). However, the radio flux density of three out of these seven optically unidentified sources is brighter than $1 \mathrm{mJy}$, significantly brighter than the sources studied by Richards et al. (1999). These sources are either extremely reddened or extremely "radio loud." They invite follow-up infrared imaging to clarify their character.

Altogether, there are 23 radio sources (counting sources 19 and 21 as a single one) for which a classification is possible based on the X-ray emission alone. Of these, 15 (65\%) have an AGN designation, four (17\%) are QSOs, and four are classified as normal galaxies. Thus, based on the properties of X-ray counterparts, $19(30 \%)$ of the radio sources have evidence of an AGN. Considering only the submillijansky radio sources in the sample, 15 out of 55 sources $(27 \%)$ show signs of AGN activity based on their detected X-ray emission alone. The proportion of faint radio sources associated with AGN X-ray sources is thus significantly higher than the proportion $(20 \%)$ of radio sources with evidence of AGN activity reported by Richards et al. (1999) on the basis of classification by radio spectral index and optical morphology.

It is claimed that very deep radio images will provide a population of radio-selected objects that are of great astrophysical interest, since they will be selected without the intervention of dust obscuration. The present survey lends weight to this claim. The optical and X-ray counterparts of the faint radio sources, as well as the blank fields, invite further study at infrared wavelengths since many will be galaxies exhibiting evidence of violent star formation and AGN activity at intermediate and high redshifts.

\section{CONCLUSIONS}

We present the cross-correlation between a deep radio survey of the GOODS CDF-S ACS field and the available optical and $\mathrm{X}$-ray data. Out of the 64 radio sources in this region, 58 have an optical and/or X-ray identification. Spectroscopic or photometric redshifts are available for the majority of these sources, which allows a classification to be established. The superb ACS imaging quality reveals the morphological characteristics of the radio sample, which can now be used, in particular, to analyze in more detail the morphology of radio-selected star-forming galaxies.

In spite of the very deep optical observations available, seven radio sources remain optically unidentified, with all but one revealing no indications of X-ray emission. Infrared observations, 
in particular those being done by the Spitzer Space Telescope, will probably reveal some of these sources, likely to be very dusty star-forming galaxies or very high redshift AGNs. Further radio observations will also be needed to clarify the physical mechanisms present in these sources.
J. A. gratefully acknowledges support from the Science and Technology Foundation (Portugal) through the research grant POCTI/CTE-AST/58027/2004. We thank the anonymous referee for insightful and constructive comments that have improved this paper.
Adami, C., et al. 2005, A\&A, 443, 805

Afonso, J., Georgakakis, A., Almeida, C., Hopkins, A., Cram, L., Mobasher, B., \& Sullivan, M. 2005, ApJ, 624, 135

Alexander, D. M., Aussel, H., Bauer, F. E., Brandt, W. N., Hornschemeier, A. E., Vignali, C., Garmire, G. P., \& Schneider, D. P. 2002, ApJ, 568, L85

Alexander, D. M., et al. 2003, AJ, 126, 539

Chapman, S. C., et al. 2003, ApJ, 585, 57

Condon, J. J. 1992, ARA\&A, 30, 575

Croom, S. M., Warren, S. J., \& Glazebrook, K. 2001, MNRAS, 328, 150

Daddi, E., et al. 2004, ApJ, 600, L127

Georgakakis, A., Mobasher, B., Cram, L., Hopkins, A., Lidman, C., \& RowanRobinson, M. 1999, MNRAS, 306, 708

Giacconi, R., et al. 2001, ApJ, 551, 624 2002, ApJS, 139, 369

Giavalisco, M., et al. 2004, ApJ, 600, L93

Gilli, R., et al. 2003, ApJ, 592, 721

Hornschemeier, A. E., et al. 2001, ApJ, 554, 742 2003, AJ, 126, 575

Le Fèvre, O., et al. 2004, A\&A, 428, 1043

Maccacaro, T., Gioia, I., Wolter, A., Zamorani, G., \& Stocke, J. 1988, ApJ, 326, 680

\section{REFERENCES}

Merloni, A., Heinz, S., \& di Matteo, T. 2003, MNRAS, 345, 1057

Mignoli, M., et al. 2005, A\&A, 437, 883

Mobasher, B., et al. 2004, ApJ, 600, L167

Richards, E. A., Fomalont, E. B., Kellermann, K. I., Windhorst, R. A., Partridge, R. B., Cowie, L. L., \& Barger, A. J. 1999, ApJ, 526, L73

Richards, E. A., Kellermann, K. I., Fomalont, E. B., Windhorst, R. A., \& Partridge, R. B. 1998, AJ, 116, 1039

Schmidt, M., et al. 1998, A\&A, 329, 495

Shapley, A., Fabbiano, G., \& Eskridge, P. B. 2001, ApJS, 137, 139

Stocke, J. T., Morris, S. L., Gioia, I. M., Maccacaro, T., Schild, R., Wolter, A., Fleming, T. A., \& Henry, J. P. 1991, ApJS, 76, 813

Sutherland, W., \& Saunders, W. 1992, MNRAS, 259, 413

Szokoly, G. P., et al. 2004, ApJS, 155, 271

van Dokkum, P. G., Kriek, M., Rodgers, B., Franx, M., \& Puxley, P. 2005, ApJ, 622, L13

Vanzella, E., et al. 2005, A\&A, 434, 53

Windhorst, R. A., Miley, G. K., Owen, F. N., Kron, R. G., \& Koo, D. C. 1985 , ApJ, 289, 494

Wolf, C., et al. 2004, A\&A, 421, 913

Yun, M. S., Reddy, N. A., \& Condon, J. J. 2001, ApJ, 554, 803

Zheng, W., et al. 2004, ApJS, 155, 73 